Historic, archived document

Do not assume content reflects current scientific knowledge, policies, or practices. 

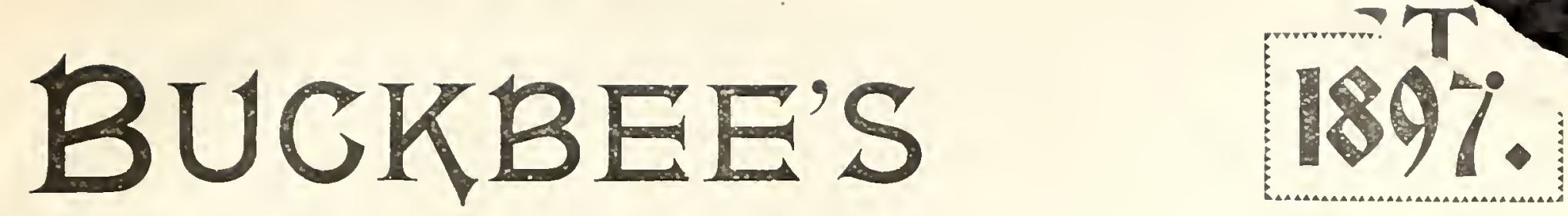

\section{Special Trade List}
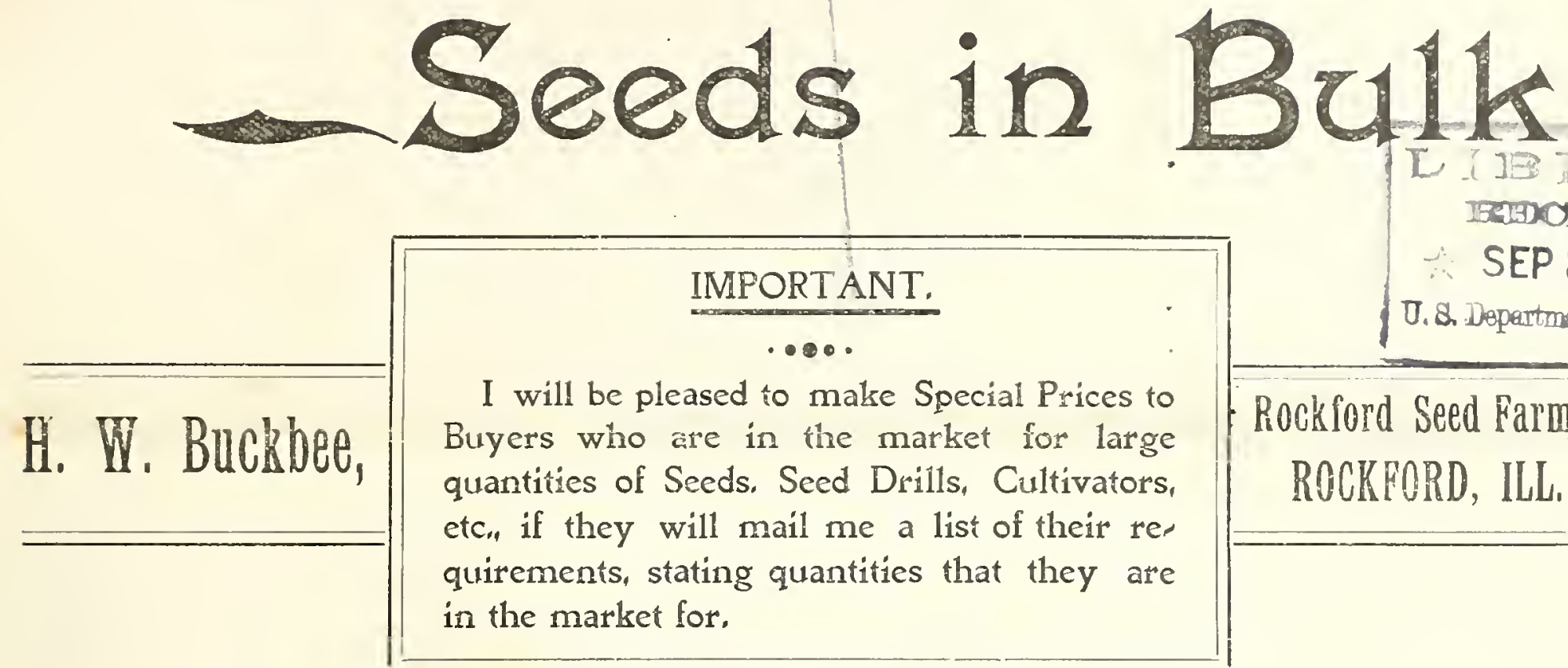

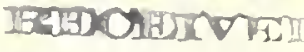

SEP $8-\sqrt{323}$

T. \&. Departmont of Agritoriticit

Rockford Seed Farms, ROCKFORD, ILL,

Not Bound by these Prices for any Definite Time or Quantity, and subject to advance without notice.

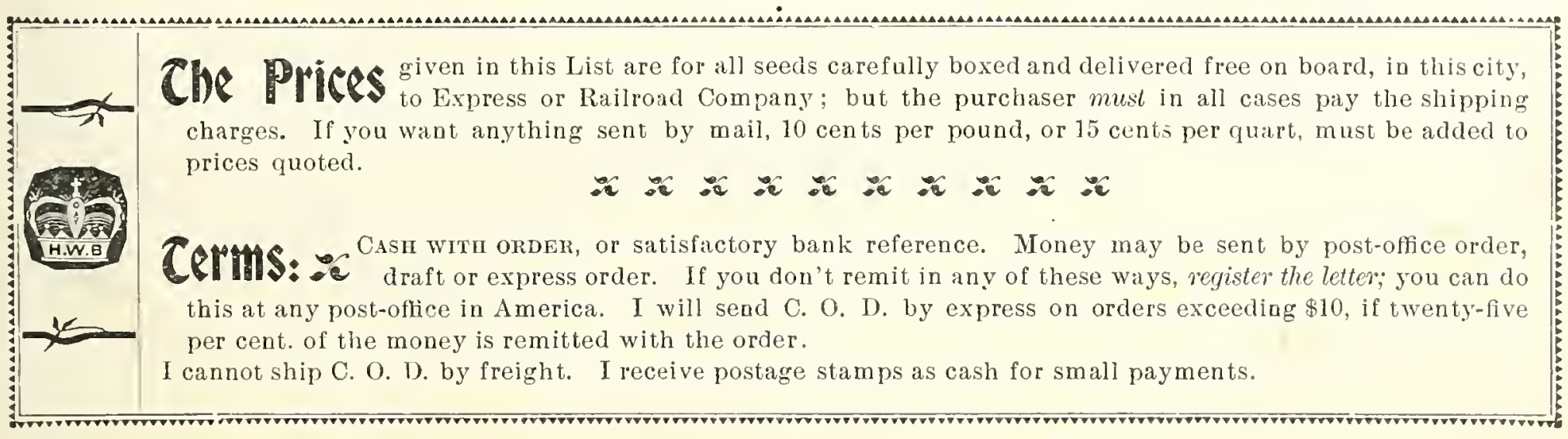

\section{BEANS.}

Buckbee's New Golden Lima, (Pkts. only-Pkt. 15c., I doz. \$1.50).

\begin{tabular}{|c|c|c|c|}
\hline thee's Improved Extra Early Valentine. & & & \\
\hline W Dwarf Horticultural or Cranberry & .15 & 65 & 2.25 \\
\hline 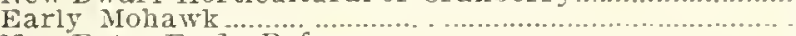 & .12 & .6 .3 & 2.25 \\
\hline New Extra Early Refugee. & .12 & .65 & 2.25 \\
\hline Early Red Valentine......... & & & \\
\hline White Valentine. & .12 & .65 & 2.25 \\
\hline Round Yellow six Weeks & & .65 & \\
\hline 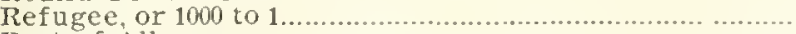 & & & 225 \\
\hline Best of All..... & .15 & .65 & 2.25 \\
\hline Improred Long Yellow Sis Weeks.. & .12 & .65 & .25 \\
\hline Buckbee's Electric Tree.. & & .90 & \\
\hline Early China Red Eye. & .15 & .65 & 2.25 \\
\hline Early Royal Dwart Kidney.. & .10 & .65 & 2.20 \\
\hline Improved Large White Marrow. & & .65 & \\
\hline Prolific Tree Bean... & .10 & .65 & 0 \\
\hline Navy Bean (select). & .10 & .6а̃ & 2.00 \\
\hline New Jackson Wonder Bush Lima & & 1.25 & \\
\hline Henderson's Bush Lima................ & .20 & 1.20 & \\
\hline Burpee's Bush Lima. & .20 & 1.20 & \\
\hline Yellow Eyed Wax. & & & \\
\hline Black Eyed Wax... & .15 & & \\
\hline Pink Eyed Wax. & .15 & .80 & .75 \\
\hline Buckbee's Rust Proof Golden Eyed Wax.. & .20 & 1.00 & \\
\hline 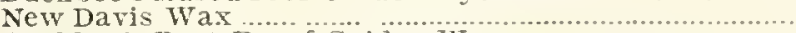 & & & 0 \\
\hline Buckbee's Rust Proof Golden Wa & .20 & 3.00 & 0 \\
\hline New Prolific German or Improved Black Wax. & .18 & .90 & \\
\hline Improved White Wax or Butter. & .18 & .90 & 0 \\
\hline New Yosemite Mammoth Wax... & .20 & 1.20 & \\
\hline ell's Kidney Wax.......... & .18 & .80 & \\
\hline New Flageolet Wax... & .18 & .90 & \\
\hline Keeney's Rustless Golden Wax & & 1.20 & \\
\hline
\end{tabular}

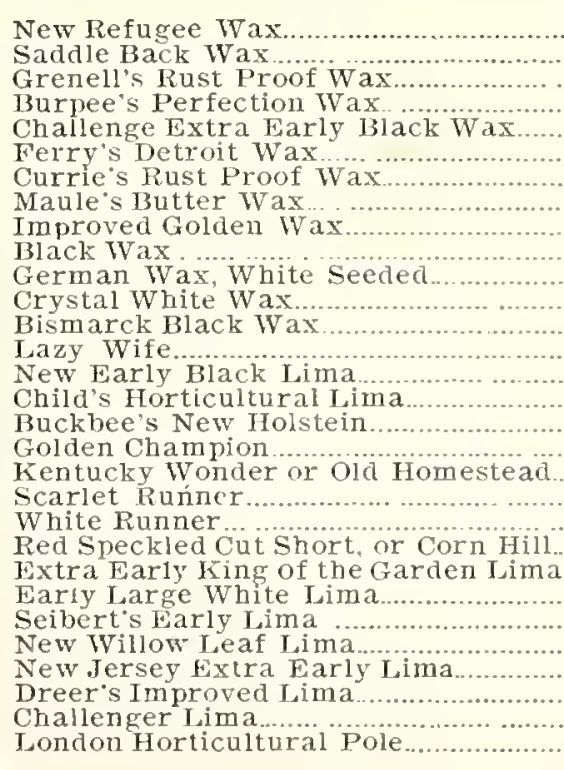


If wanted by mail, add io cents per pound or I 5 cents per quart to prices quoted, to cover postage.

SWEET CORN-Selected.

\begin{tabular}{|c|c|c|c|}
\hline lirst of All......... & & & \\
\hline Buckbee" "Private Stock" Evergreen... & & & (3.. \\
\hline 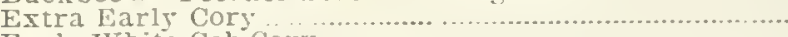 & &. & \\
\hline $\mathrm{Cob}$ Cory & & & \\
\hline Adams & & & \\
\hline esota. & & & \\
\hline arly Chicago Warket... & & .40 & \\
\hline 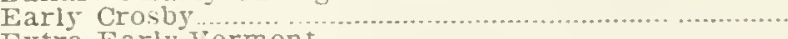 & & & \\
\hline erm & & & \\
\hline$a x+y . . .$. & & & \\
\hline $\mathrm{Bu}$ & & .50 & \\
\hline & & & \\
\hline & & & \\
\hline & & & \\
\hline & & 0 & \\
\hline & & & \\
\hline & & & \\
\hline & & & \\
\hline , $\mathrm{c}$ & & & \\
\hline ........ & & & \\
\hline & & & \\
\hline & & & \\
\hline . & & & \\
\hline & & & \\
\hline e Peg & & & \\
\hline 列 & & & \\
\hline ..... & & & \\
\hline & & .6 & \\
\hline 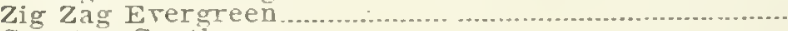 & & & \\
\hline (n...................... & .10 & & \\
\hline 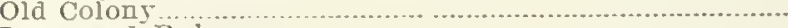 & & & \\
\hline & & & \\
\hline & & & \\
\hline & & & \\
\hline & .10 & & \\
\hline & & & \\
\hline
\end{tabular}

POP CORN

Buckbee's 'Snowball

Mapledale Prolific..

Queen's Golden.

New Red Beauty

FIELD CORN-IIY 0wn Growth.

Buckbee's New Colossal ....

Buckbee's Great Western Yellow Dent

Buckbee's Great Western Yellow Den

Western Yellow Dent.

\section{ARTICHOKE}

Large Green Giobe... ARTCHOKE'S (TUBERS.)
ART

Buckbee's Great Bonanza

Colossal........

Jerusalem

ASPARAGUS

Columbian Mam

Donald's Elmir

Barr's Mammoth

Barr's Mammoth

\section{ASPARAGUS ROOTS}

New Columbian Mammoth White

Conorer s colossa

New Palmetto

Barr's Mammoth..

\section{BEET.}

Buckbee's Sunset .......................

Improred Extra Early Eclipse.

Edmands Early Blood Turnip

Lentz Blood Turnip.

Crosby's Early Egyptian.

Detrolt Dark Red urnip...

Bastain's Blood Turnip

New Electric..............

Improved Late Blood Turnip.

Buckbee's Improved Long Blood

Buckbee's New Half Long Blood
Buckbee's Early Egyptian Blood Turnip.

Columbia

The Bismarch

Black Queen ................

Early Egyptian Blood Turnip.

\section{BEETS-Mangels.}

Buchbee's Mastodon

$20 \quad 1.25 \quad 4.00$

$.201 .25 \quad 4.00$

$\begin{array}{lll}20 & 1.25 & 4.00\end{array}$

$.0001 .25 \quad 4.00$

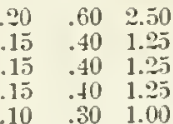

$1 / 41 \mathrm{~b} . \mathrm{ib}$

\&.75 \$2.50

$250 \quad 1000$

$.50 \quad 1.50$

$\begin{array}{ll}40 & 1.00 \\ .40 & 1.00\end{array}$

$.30 \quad .75$

$1 / 41 \mathrm{~b} .1 \mathrm{~b}$.

$\begin{array}{ll}.15 & .50 \\ .15 & .50 \\ .10 & .25 \\ & .15\end{array}$

$100 \quad 1000$

$.60 \quad 4.50$

600

$\begin{array}{ll}.50 & 3.00 \\ .60 & 4.50\end{array}$

$1 / 41 \mathrm{~b} .1 \mathrm{~b}$.

Giant Holstein

Buckbee's Mammoth Long Red.

New Golden Tankard...

Golden Mon arch ........

Champion Red Globe

New Imperial Sugar

Buckbee's Extra Early Large Jew Queen.

Extra Early Express

Extra Early Express
FIELD CORN-Continued.

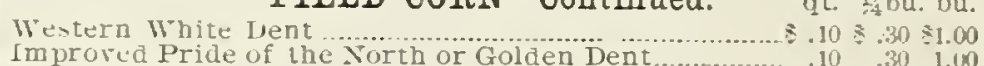

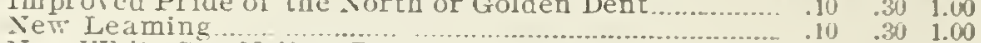

New White Cap Yellow Dent.

Chester counts yammoth

lowa Gold Mine

Boole County Prolific

Clark's Nen Early Mastadon

Tr.kory hing

Tre tra Early Large Yellow Yankee

True Thoroughbred Rural white Flint

Bweet or Sugar Corn for

\section{PEAS.}

Buckbee's Lightning Expre.

Buckbee's Selected First and Best.

Extra Earl Philadelphia

Buckbee's Extra Early Tom Thumb

'True American wonder

McLean's Little Gem ............

Nott's Excelsior

Grant's Farorite.

Horsford's Varket Garden

Bliss " New Everbearing

Telephone

Admiral (fine)

Shropshire Hero

Pride of the Marke

Sorkshire Hero

Improred Champion of England

Improred Stratagem

Large White jarrowfat

Large Black Eye Marrowfat

Improved Fjeld

Carters Firsi Crop

Extra Early Dan O'Rourke

Early May

\section{CABBAGE-Continued.}

Early Peerless.

Early Dwarf Flat Dutch

Vandergaw or Nidsummer.

Henderson's

Hendercon's Earls Sprin

New Dutch winter, or Hollander (true)

Buckbee's Great Dane

The Lupton [true]

True Early Jerser Wakefiel

Extra Early Winningstadt.

Henderson's Early Summer

Gregory's All season

Burpee's Surehead

Fottler's Improred Brunswich

Solid South

Stone Mason Marblehead

Large Late Drumhead

Autumn King

Buckbee's Mammoth Late Flat Dutch

Frotscher's Superior Late Flat Dutch

Mammoth I

Improved Large Late Flat Dutch

Premium Late Flat Dutch.

Acme Large Late Flat Dutch

Red Dutch or Pickling

Perfection Drumhea

Improved American Saroy

\section{CARROT}

Buckbee's New Danvers

Improred Intermediate...

Improred Long Orange............

Ox Heart or Guerande

Buckbee's Earliest Forcing.

Half Long Danvers.

St. Taller

Long Red Surrey

Long Red Altringham.

Early Scarlet Horn.

Halt Long Scarlet Nantes.

Buckbee Majestic

Buckbee's rictoria.

Buckbee's Improred Short White

Mammoth White Belgian

Mammoth White Belgian

CAULIFLOWER

$161 \mathrm{~b} .1 \mathrm{~b}$.

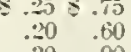

Buckbee's Extra Early Chief
Buckbee's Improred Early

Buckbee's Im proved Farls Farorite, fine

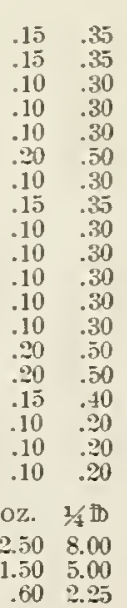

$\begin{array}{ll}.50 & 5.00 \\ .60 & 2.25\end{array}$ 


\section{Special Wholesale

If wanted by mail, add Io cents per pound or I 5 cents per quart to prices quoted, to cover postage.

CAULIFLOWER-Continued.

Henderson's True Early Snow

Early Snowbati, good stock

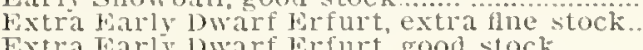

Extra Early Paris

Le Normand's Short steu

\section{CELERY}

New Golden Sulf blanching, fime.

Buckbee

New Giant Pascal

New Tíalamazoo

New liose

Perfection Heartweil

Improted Whice Pluure, new

Boston Market

Craw tord"s Half D

Celeriac or Turnip-Rooted Celery

\section{CUCUMBER}

Buckb-e's New Rockford Pickle

Buckbee's Monarch Whit
Buckbee's Internatioual.

Thorburn's New Everbearing

Buckbee's Improved Long Green

Early Russian

Garly Ginste Short Green

Early Cluste

Nichol's Iedium Green

Boston Pickling or Green Prolific

Arlington Improved White Spine

Peerless $W$ hite spine.

Evergreen White Spin

New Japanese Climbing

New Parisian Prolific Pickling

\section{EGG PLANT}

Buckbee's Improved Large New Xork Purple Extra Early Long Purple

Black Pekin

Buckbee's New White

Large New York Purple

\section{LETTUCE}

Buckbee's Superb.

New Iceberg.............

Improved Black Seeded Simpson

arigronet

Buckbee's Earliest Forcin

Simpson's Early Curled.

Early Tennis Băll, White Seeded

Early 'Tennis Ball, Black Seeded ...

Improyed Hanson

Dwarf White Heart, or Celery

New Xork Market.

Denver Market

Gold Nugget

Paris White

Buckbee's Ice Drumhead

Earty Prizehead.

Silver Ball...

Grand Rapids

Mammoth Black Seeded Butter

MUSK MELON

New Triumph

Western Giant........

Banana (Selec

New Green Fleshed Osage

White Japanese

New Banquet.

Newport

cong Persian or Casaba

Baltimore, or Acme

Improved Cantaloup

Extra Early Gran

Henderson's Perfected Delmonico

Cosmopolitan

Montreal Marke

True Tip

Golden Netted Gem

Buckbee's Ideal

New Early Hackensack......

Beck's Tew Columbus.

Buckbee's New Prolific Nutmeg

Chicago Market.

Princess

Champion ilarket

Early Nutme

Miller's Cream

Pineapple

WATER MELON.

Buckbee's Monte Cristo

Fordhook Earl

Phinney

Buckbee's Ice Cream, or Peeriess

Georgia Rattlesnake or Gypsy.

Black Spanish

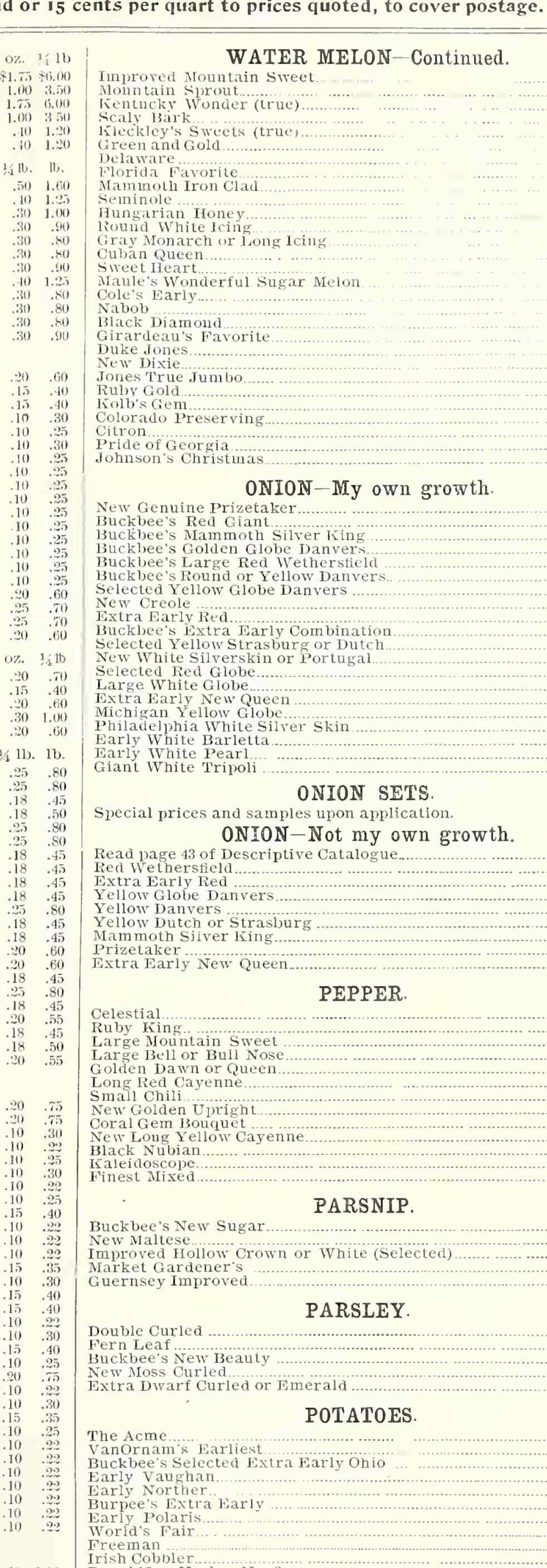

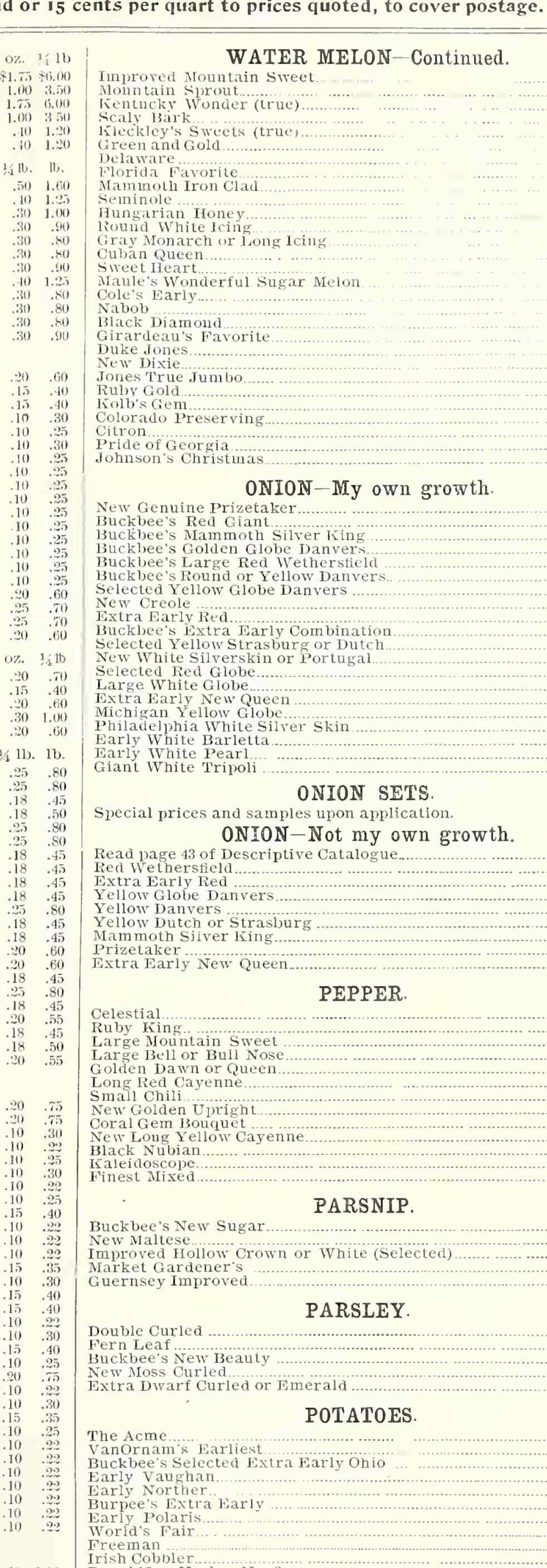

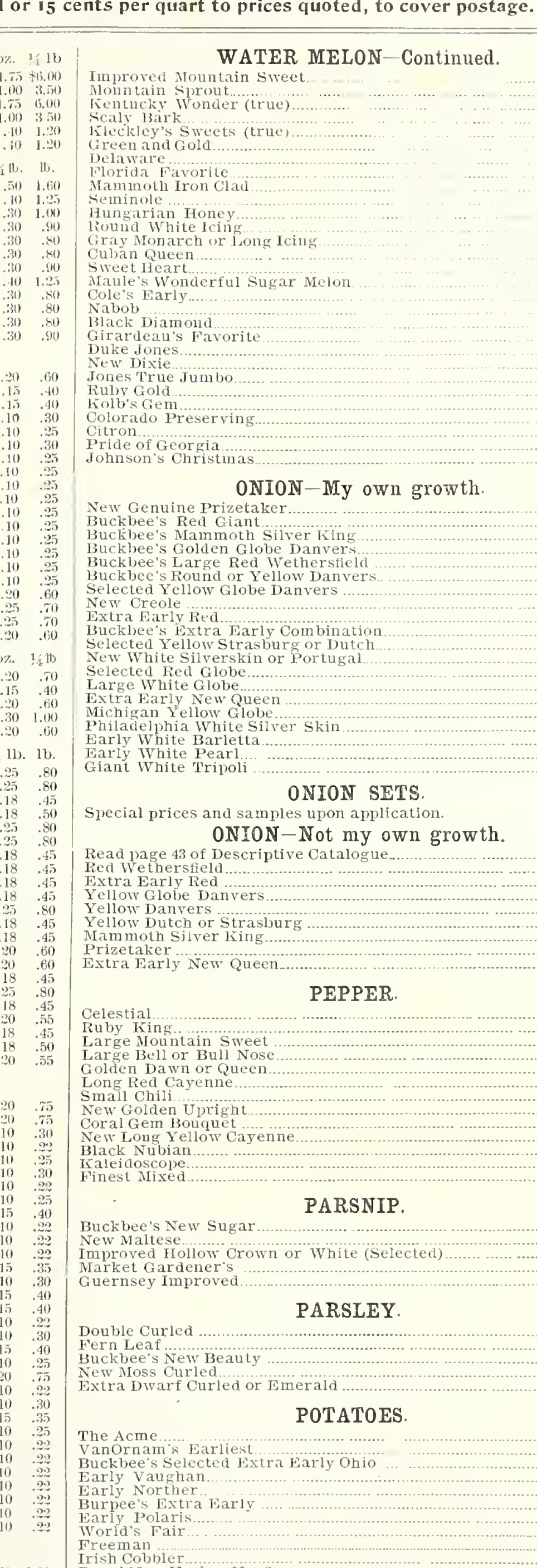

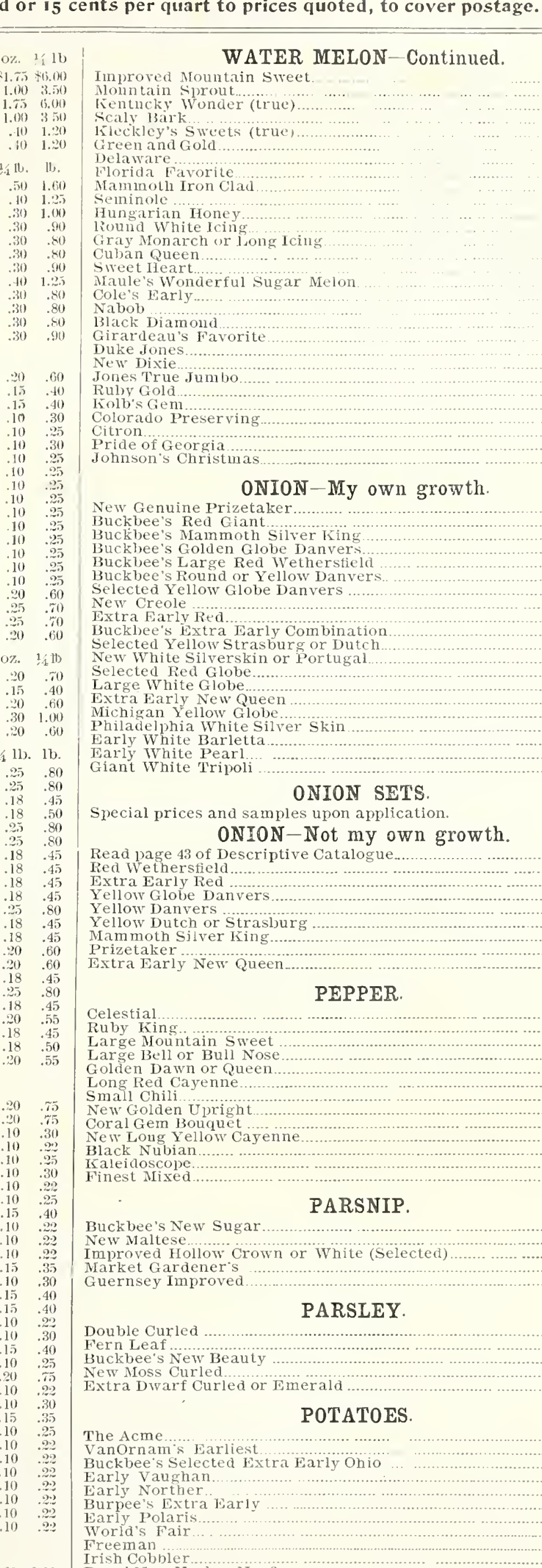

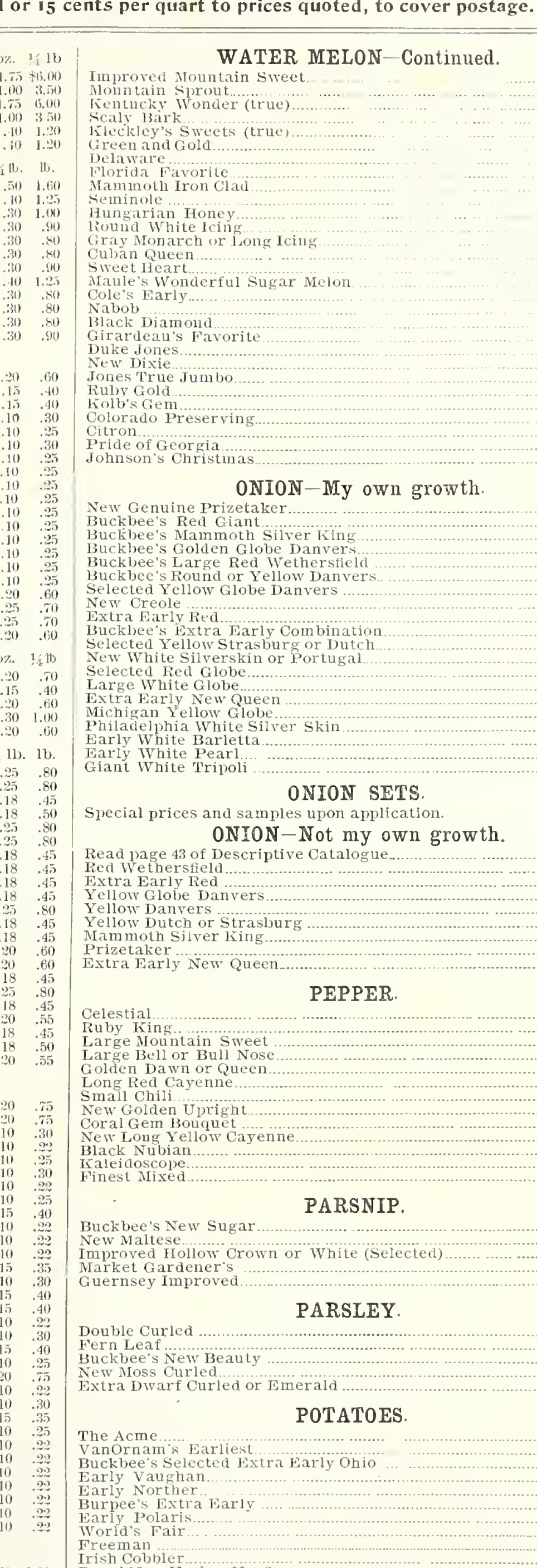

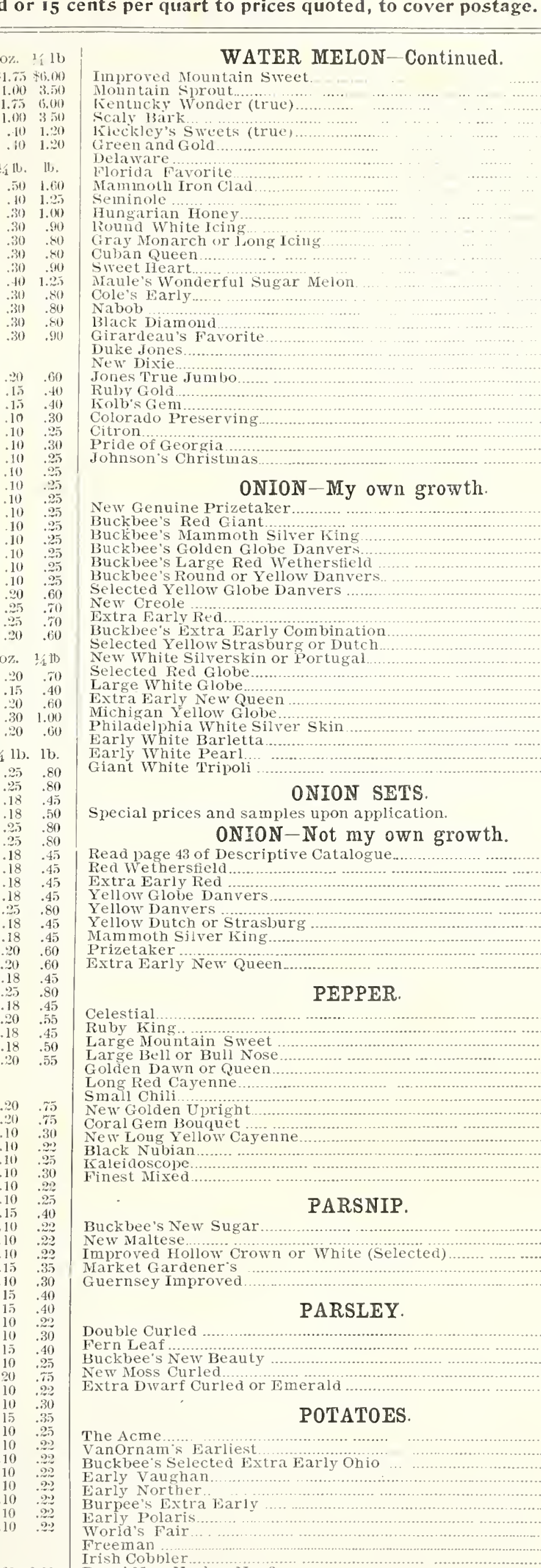

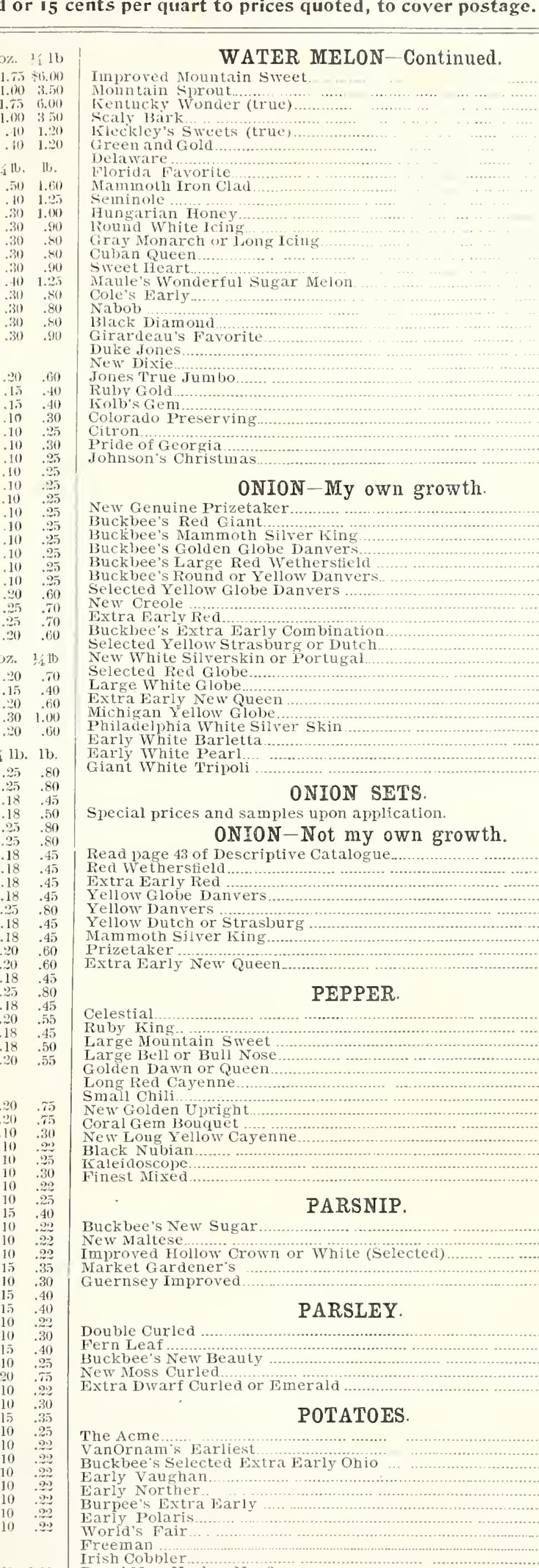

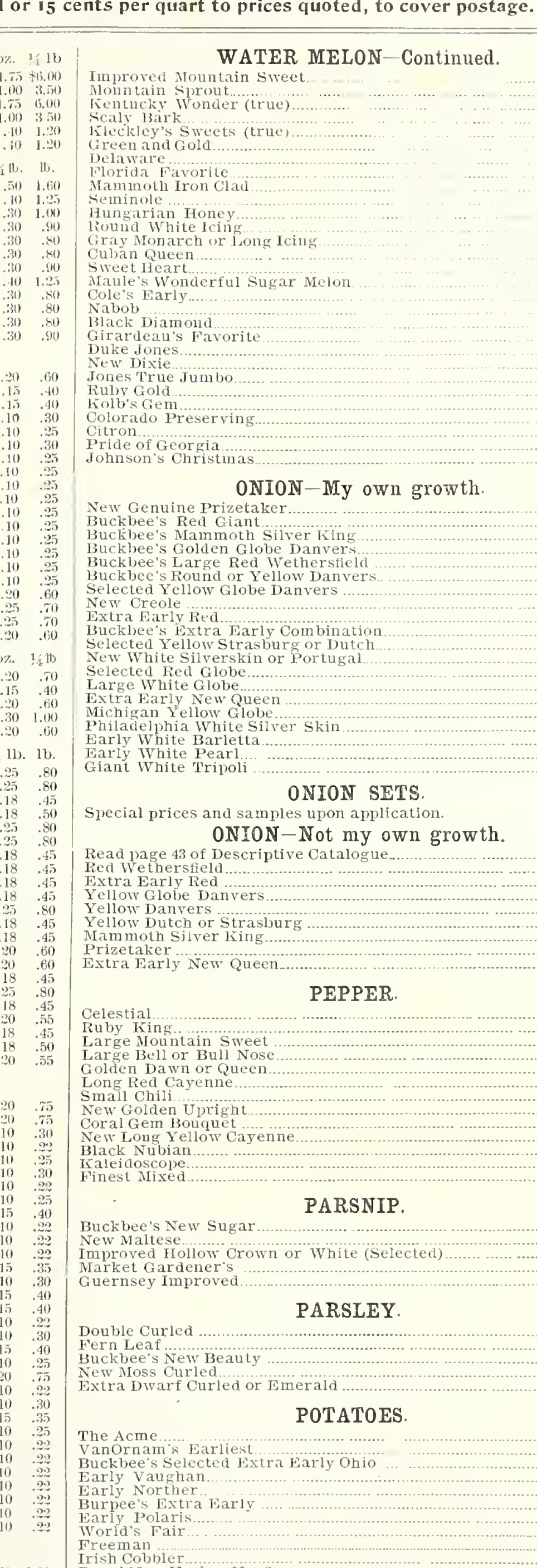

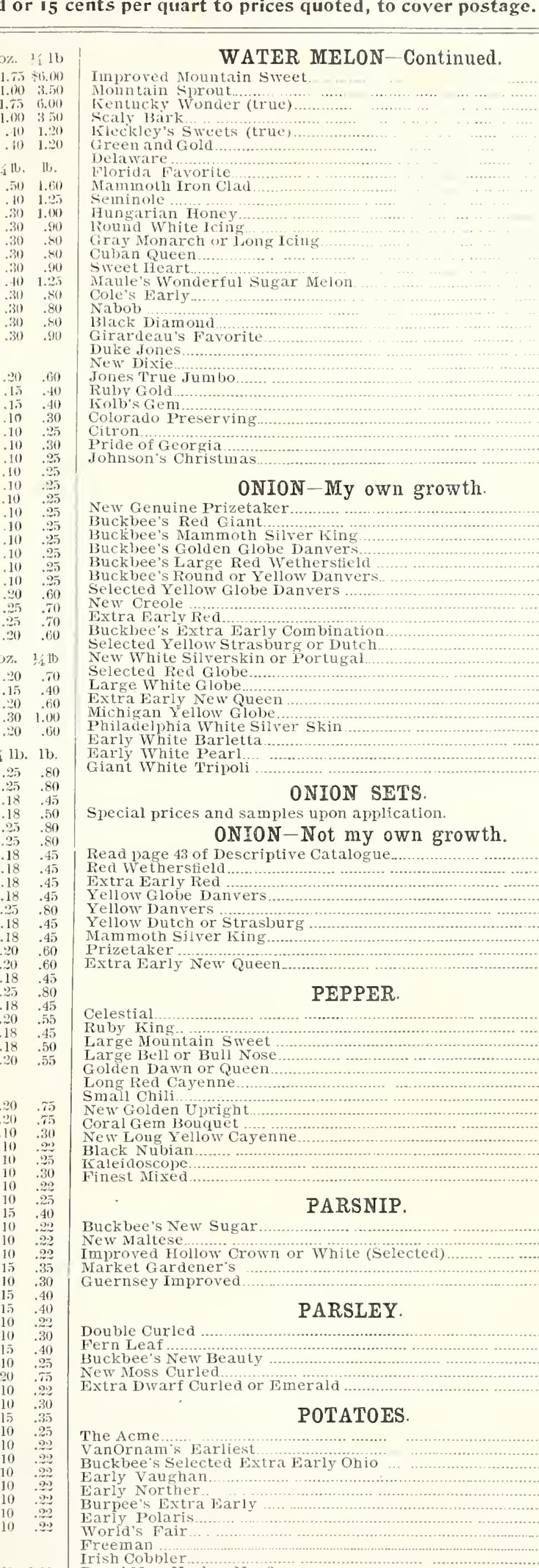

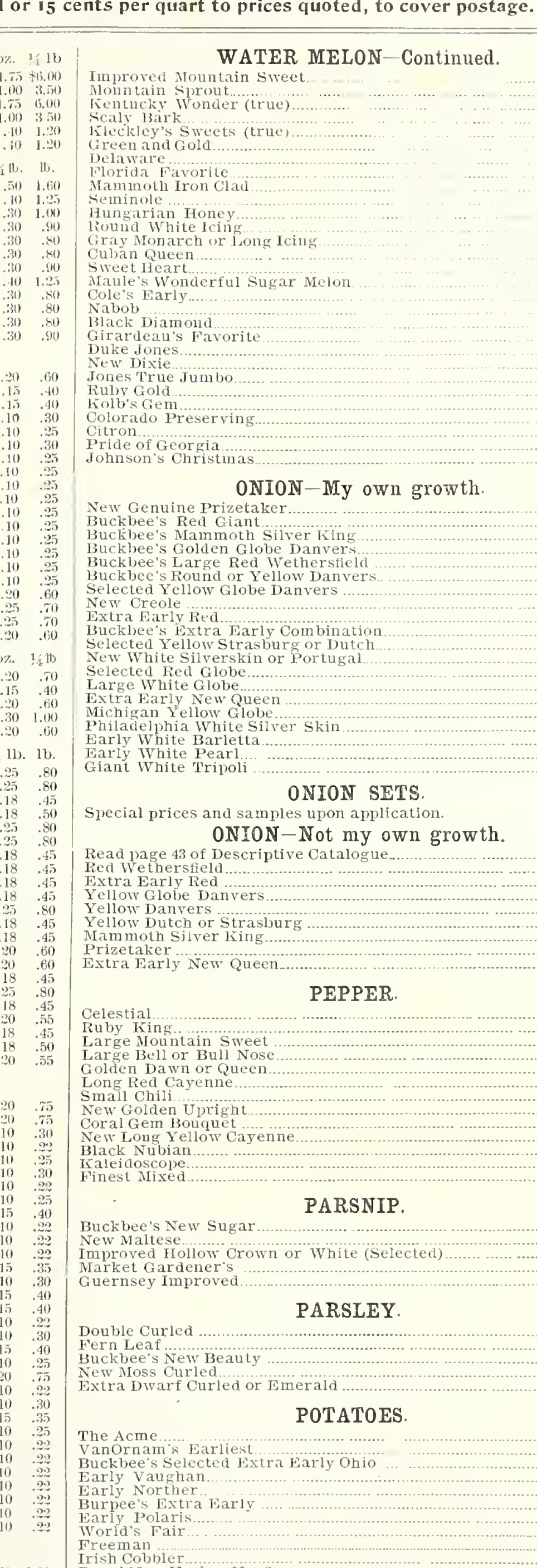

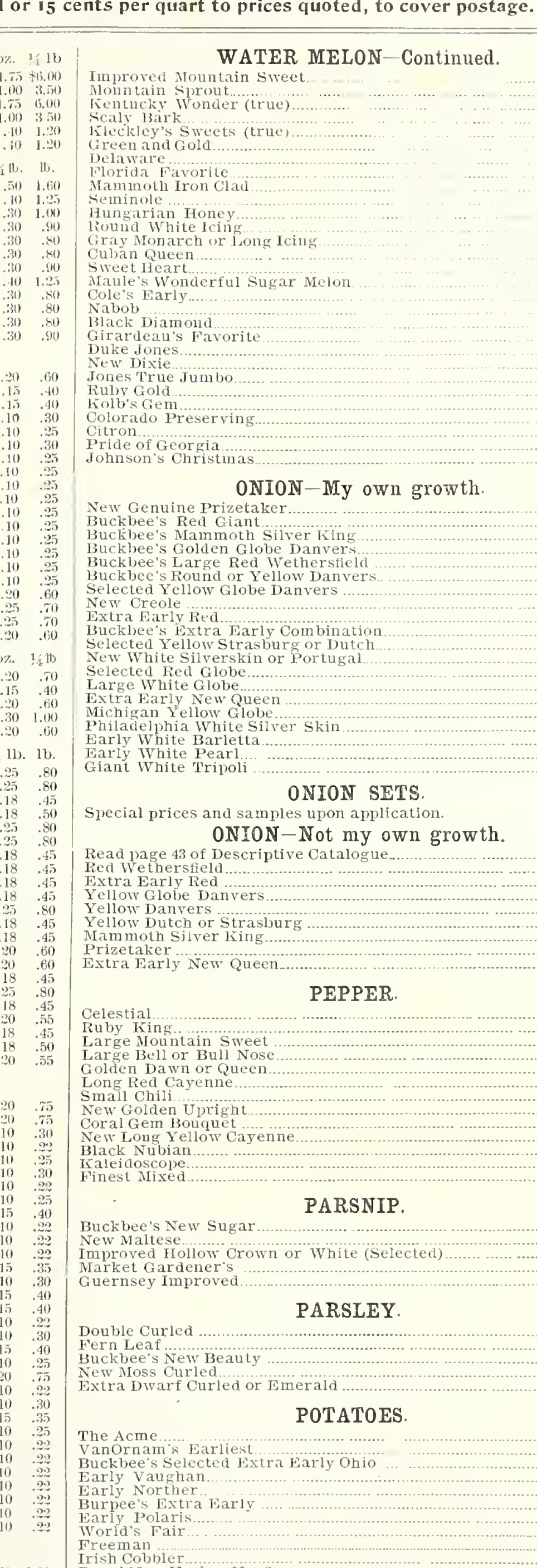

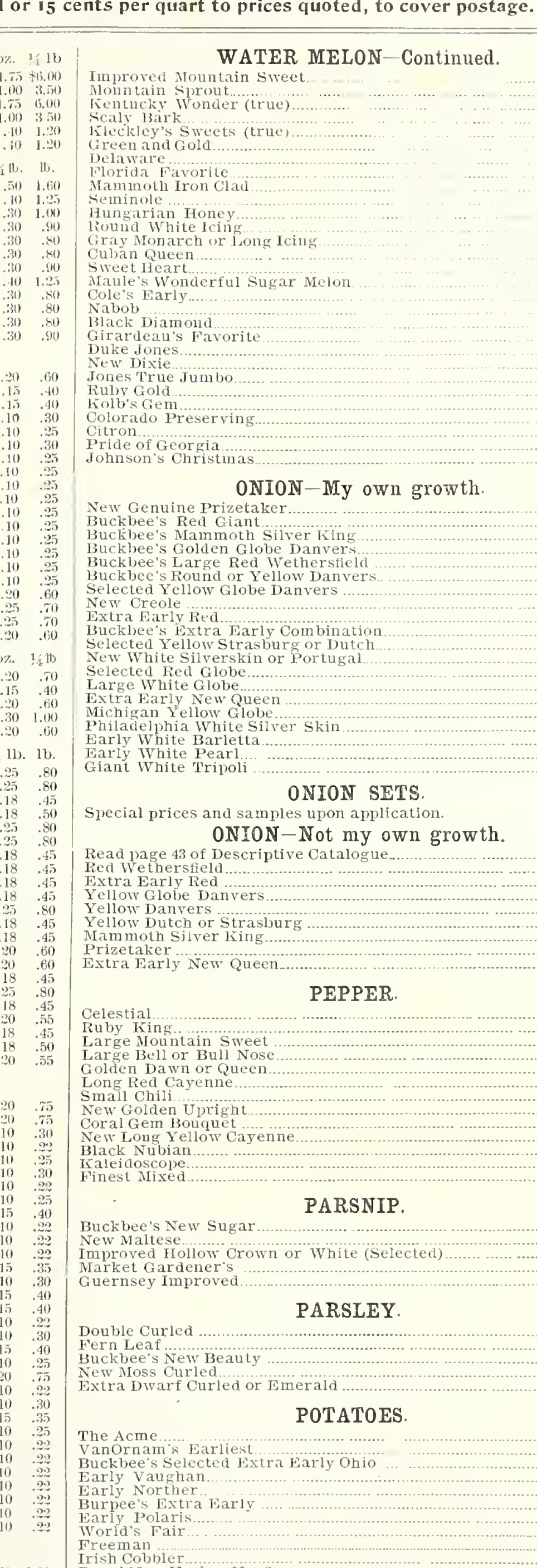

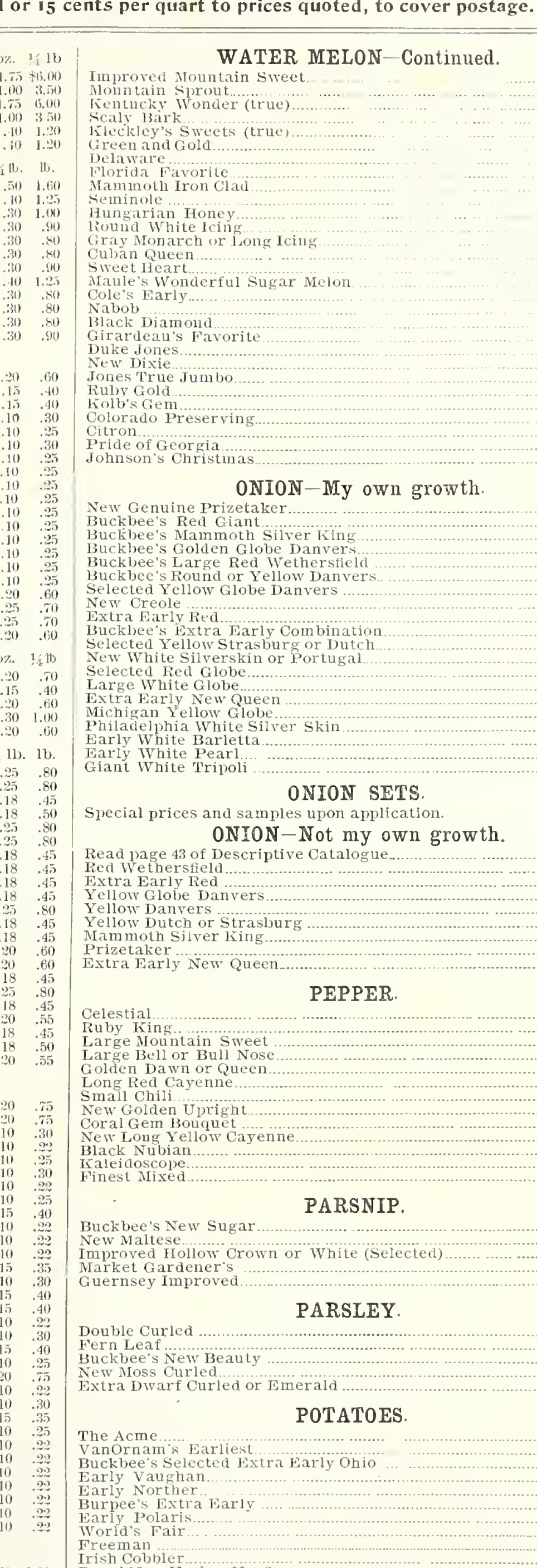

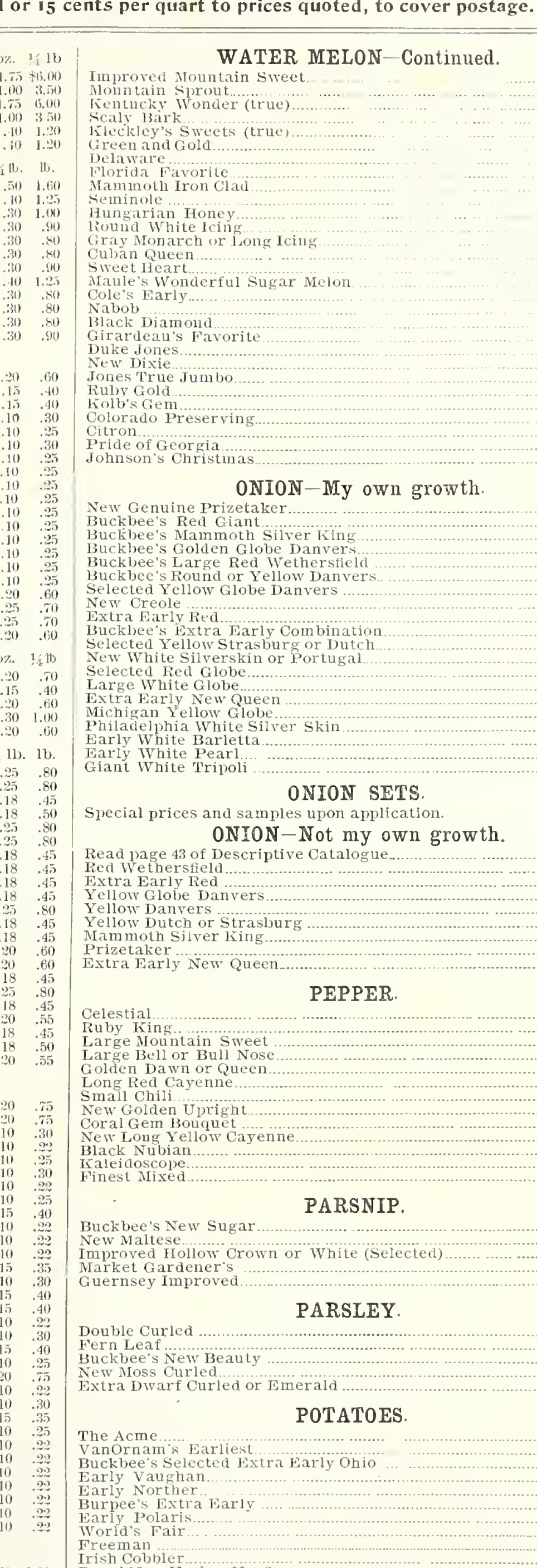

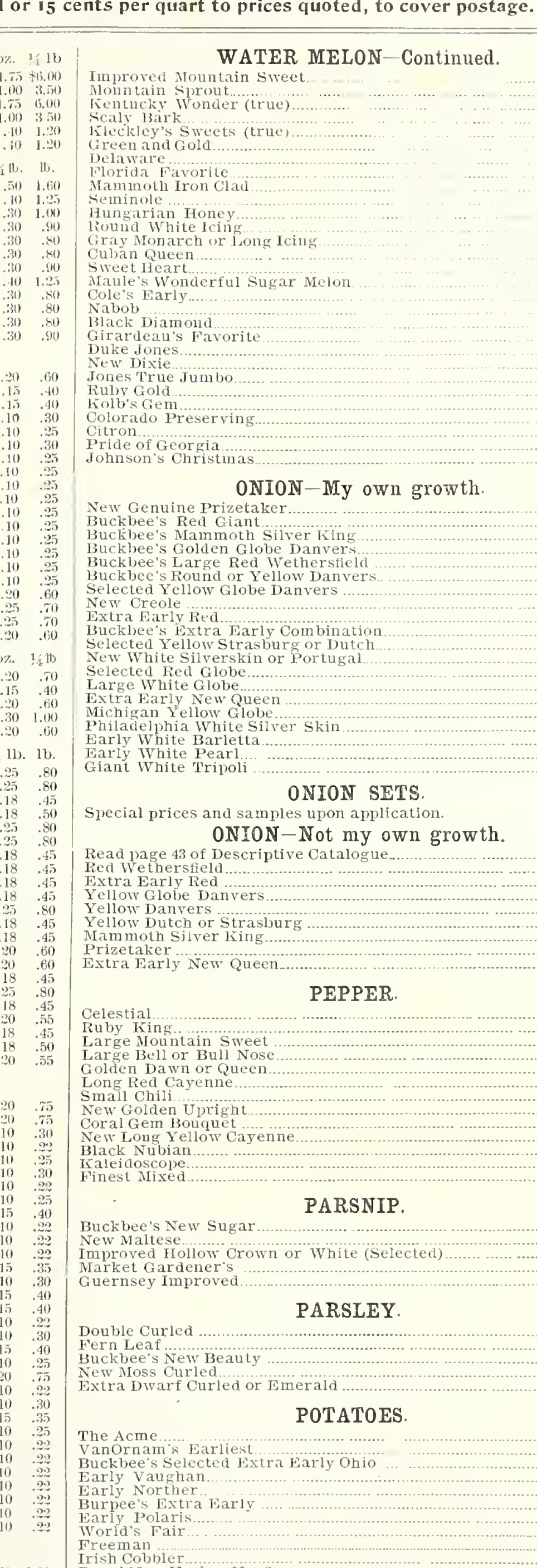

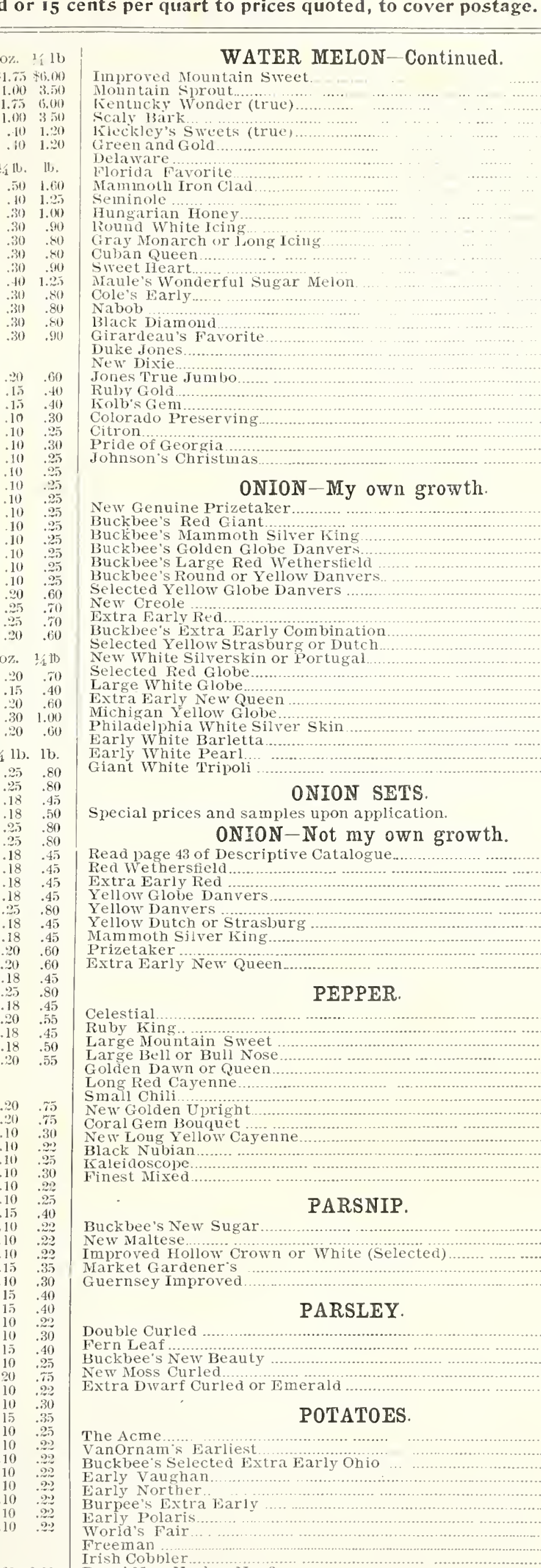

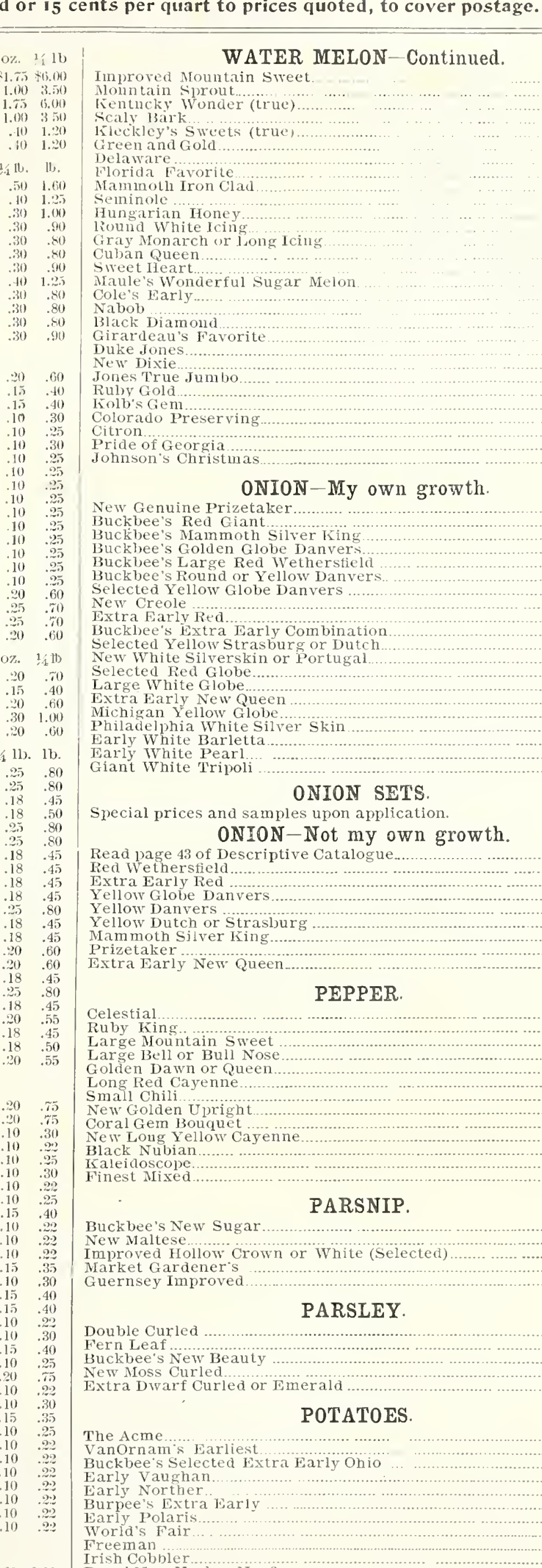

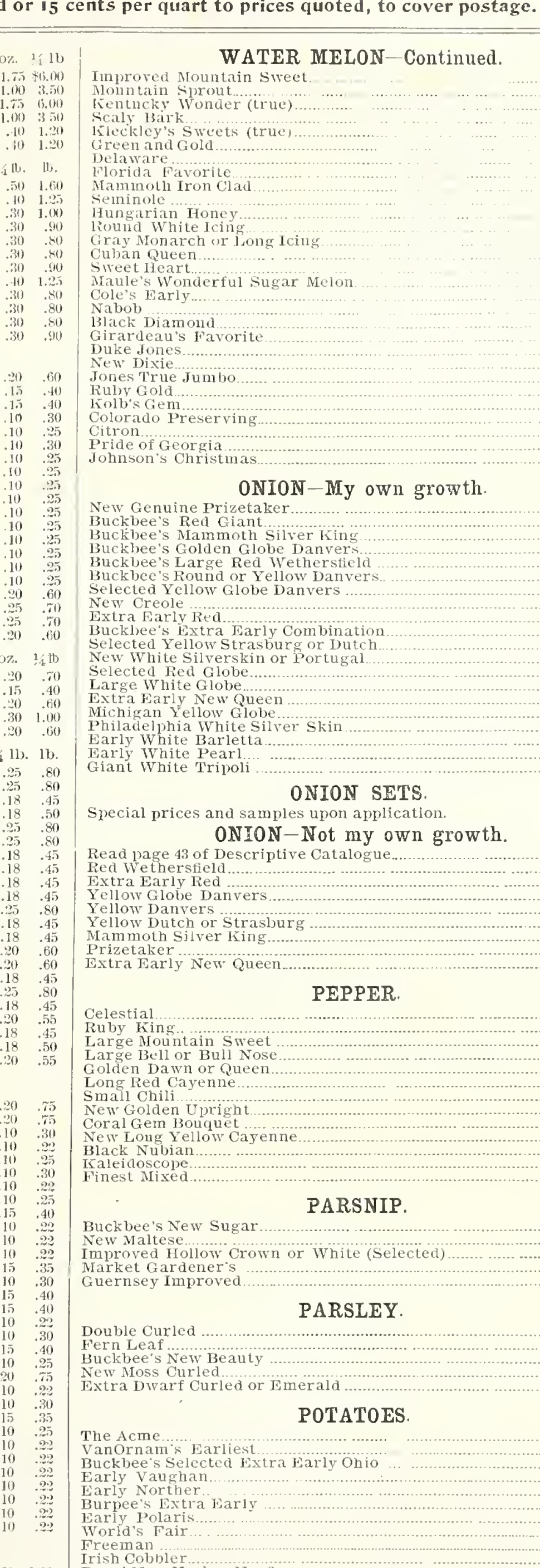

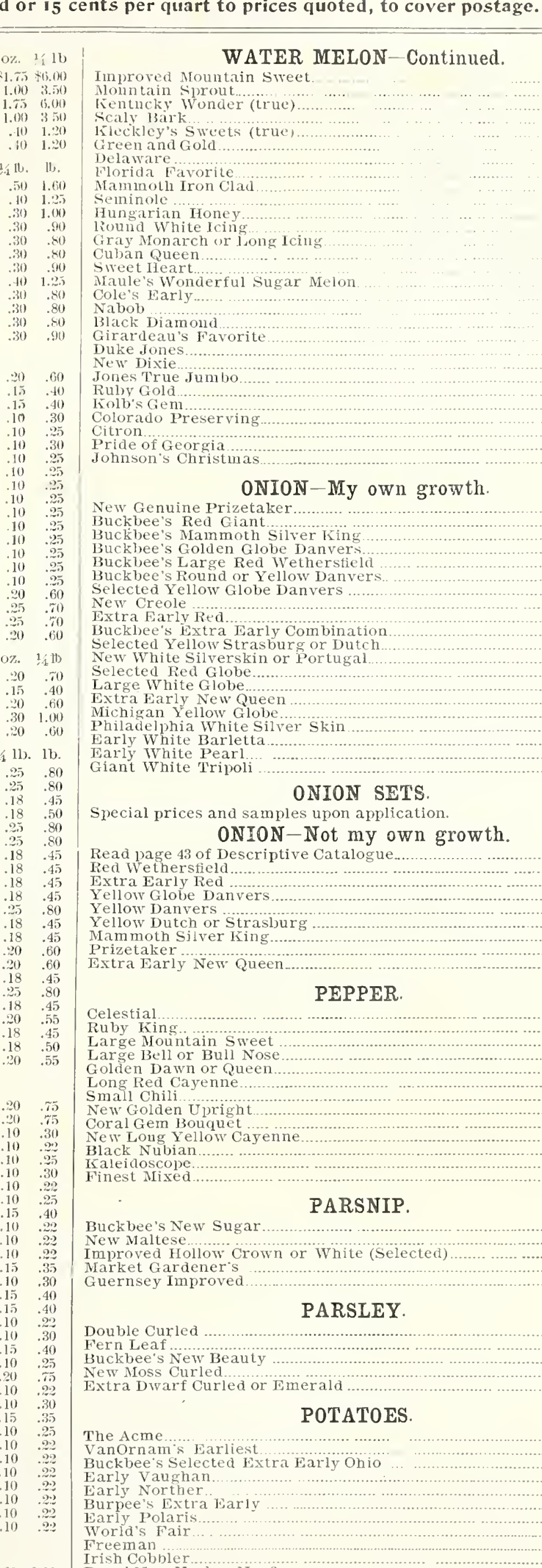

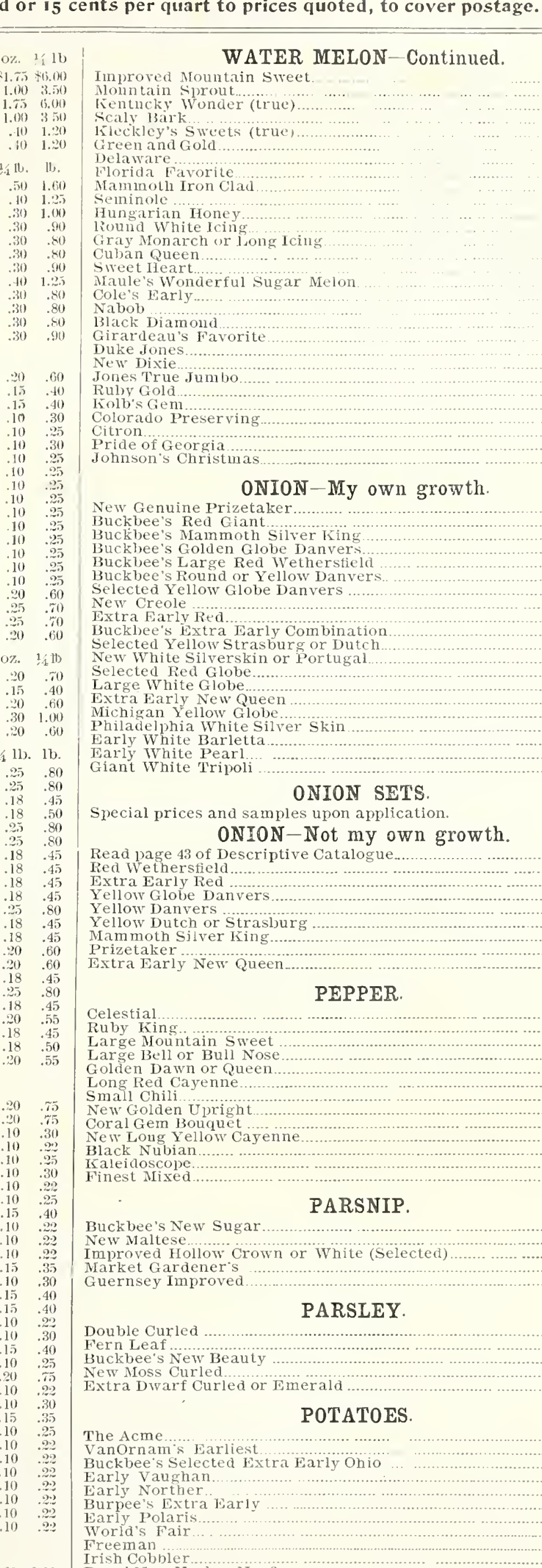

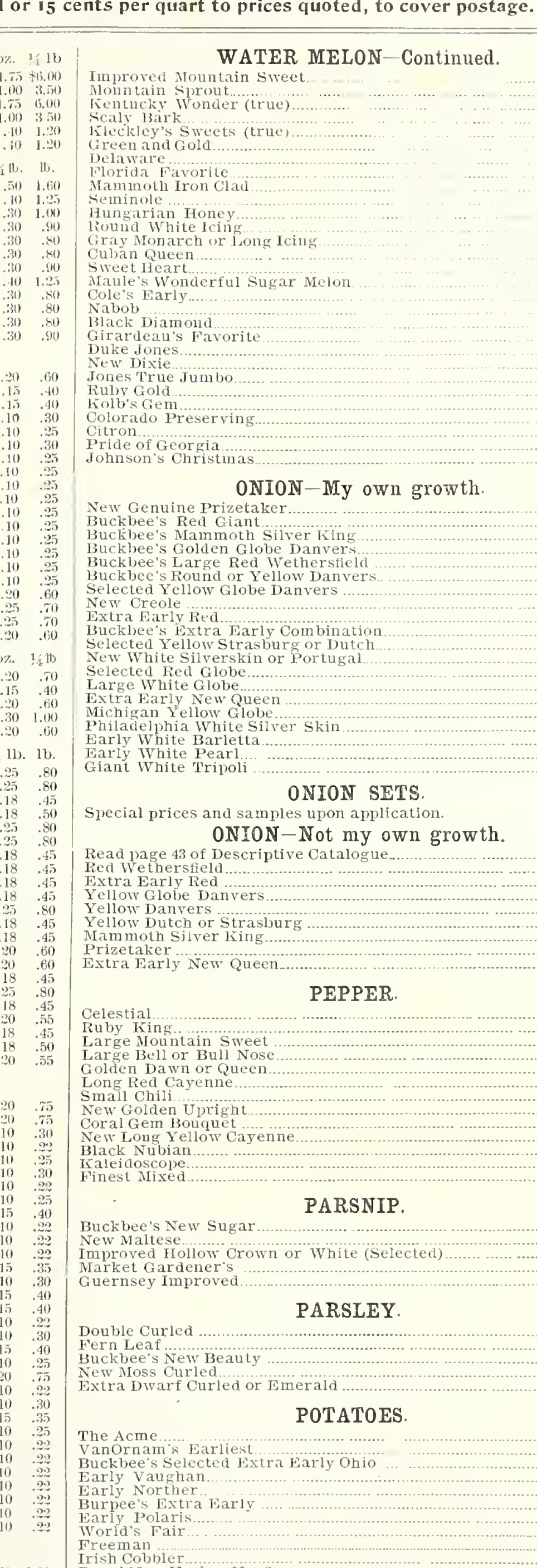

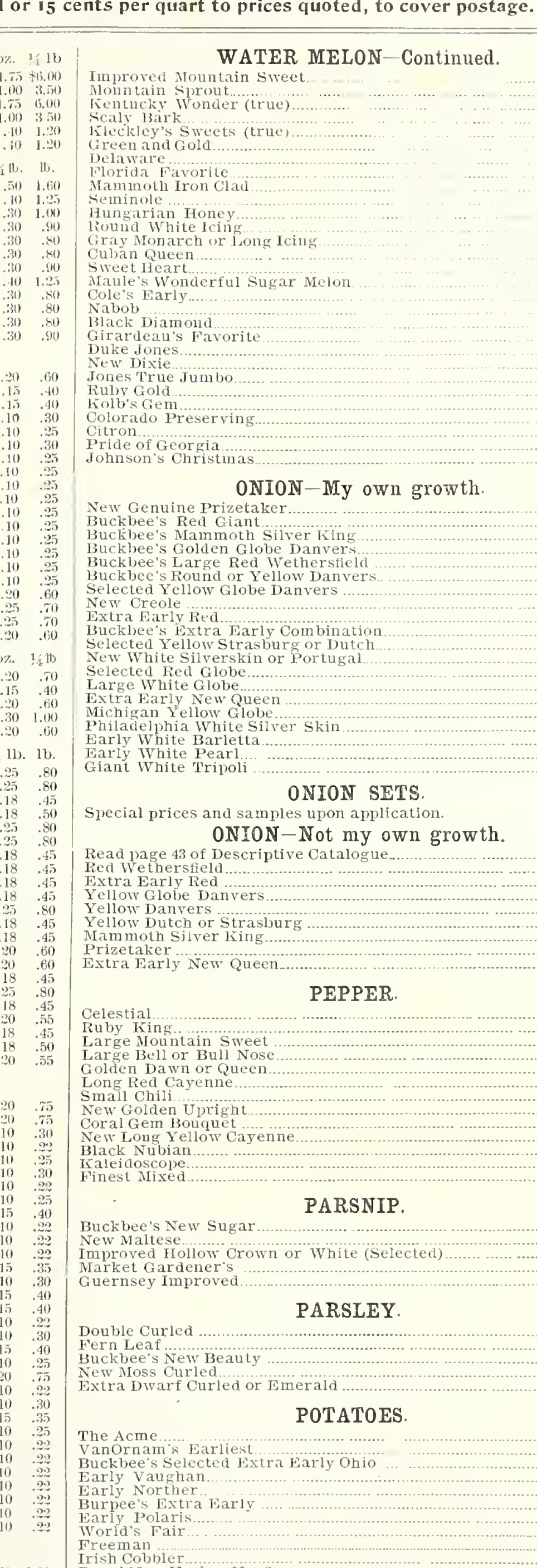

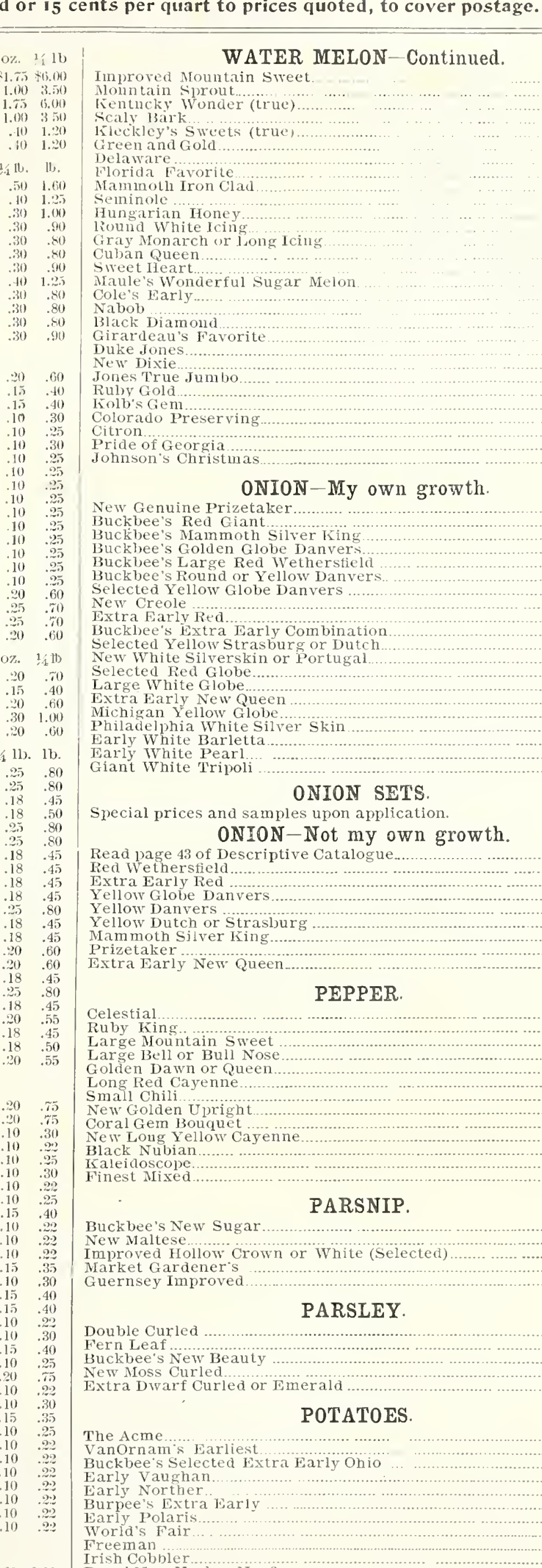

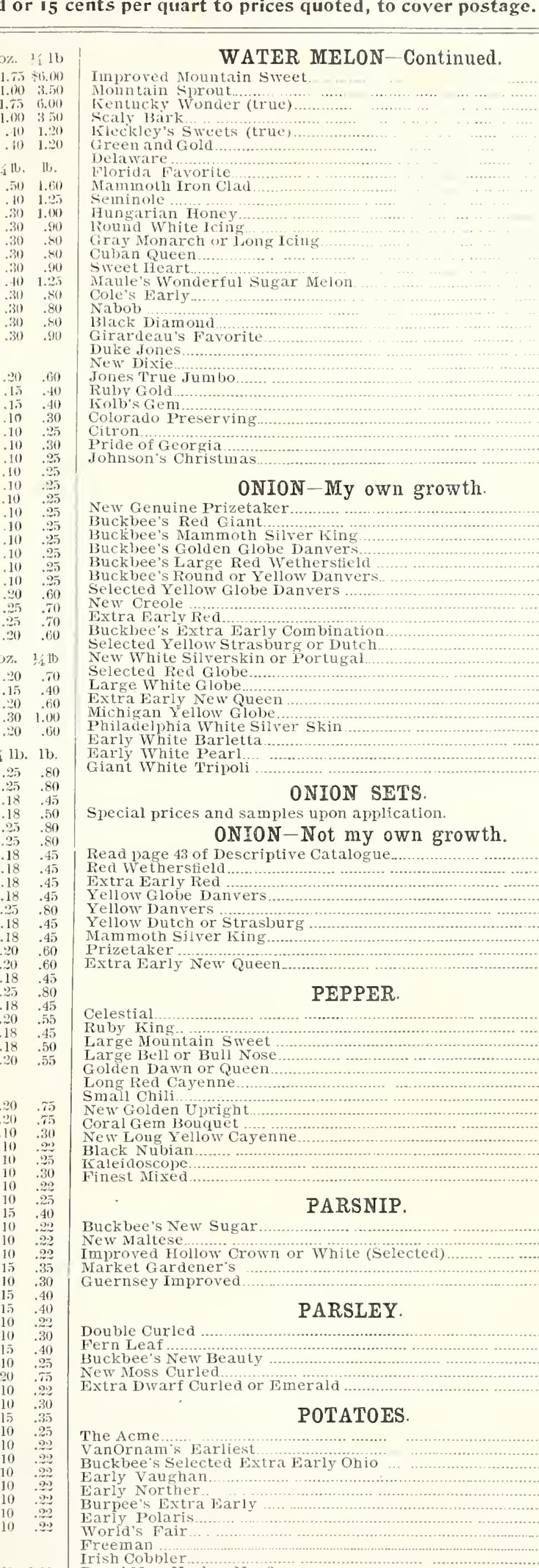

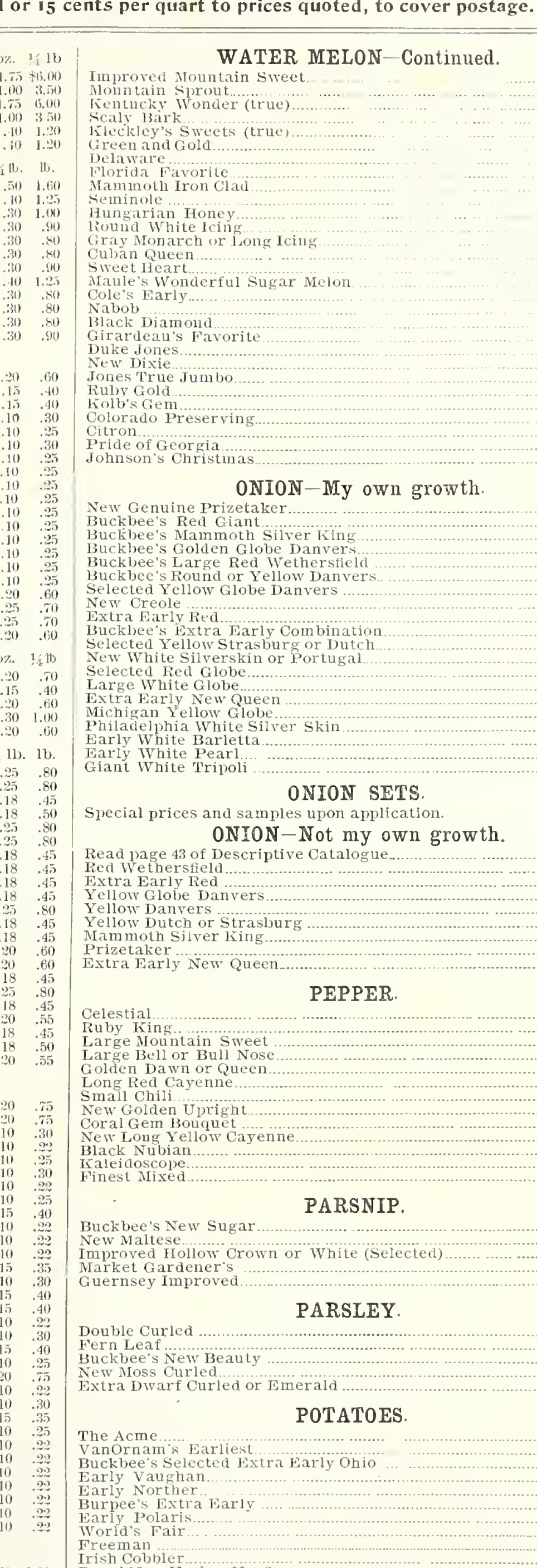

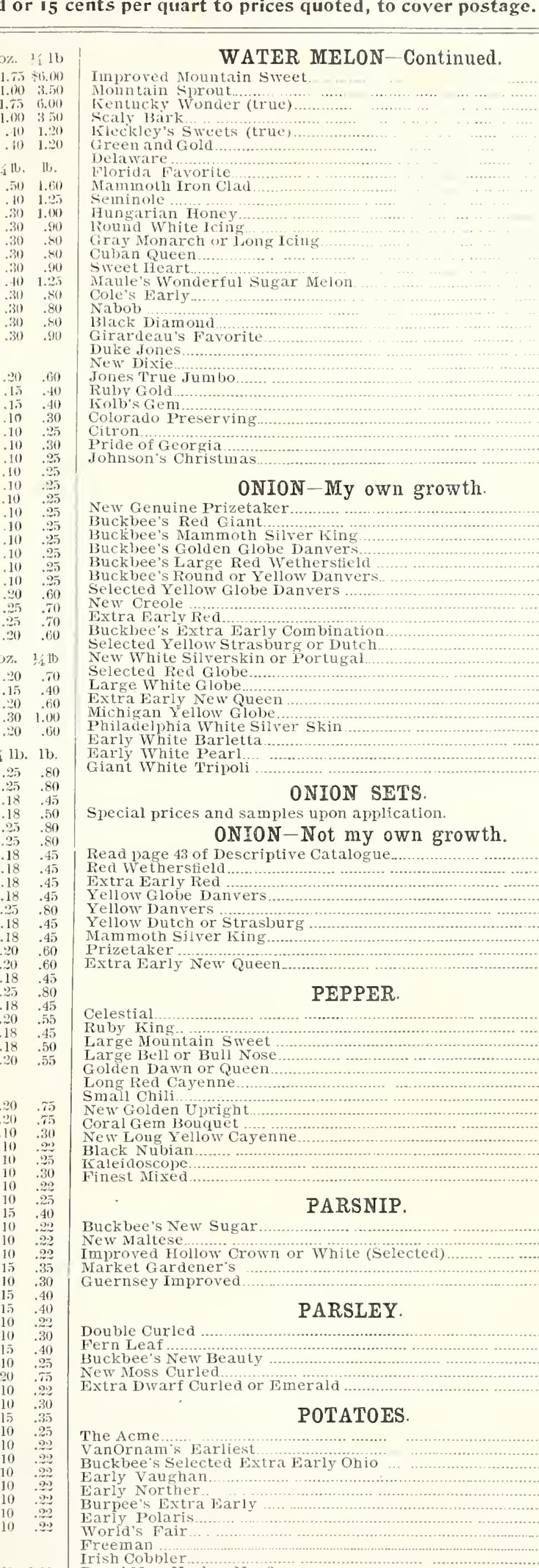

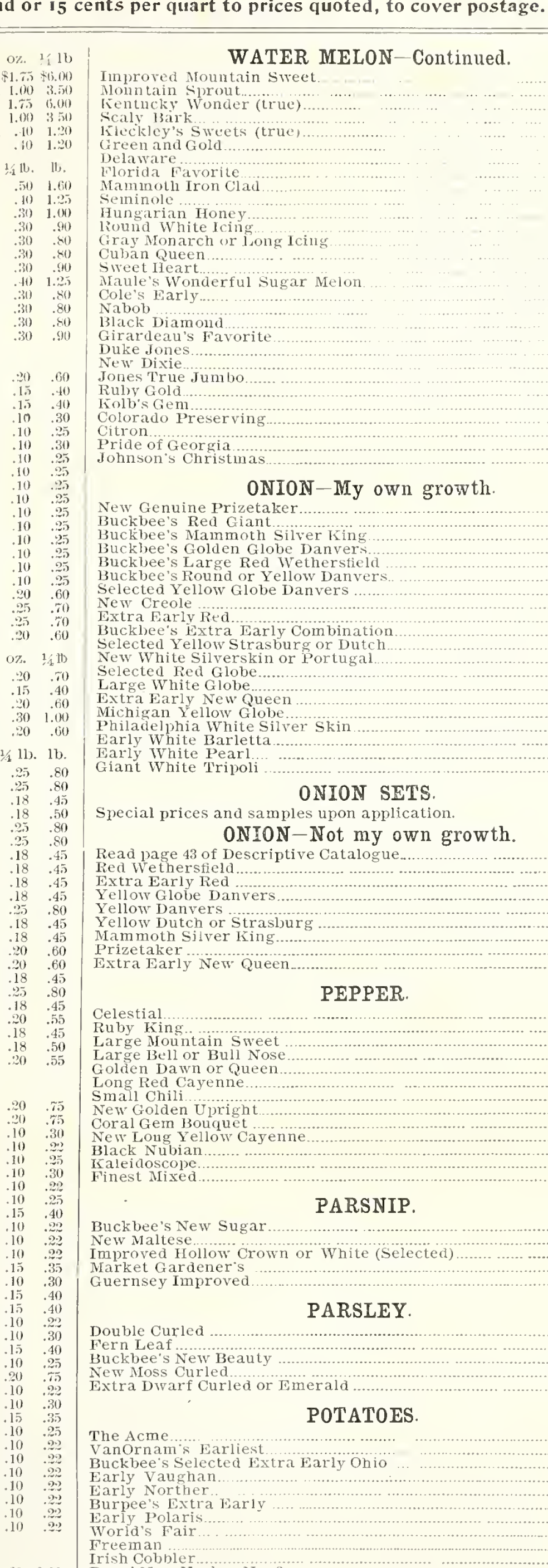

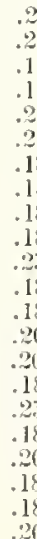

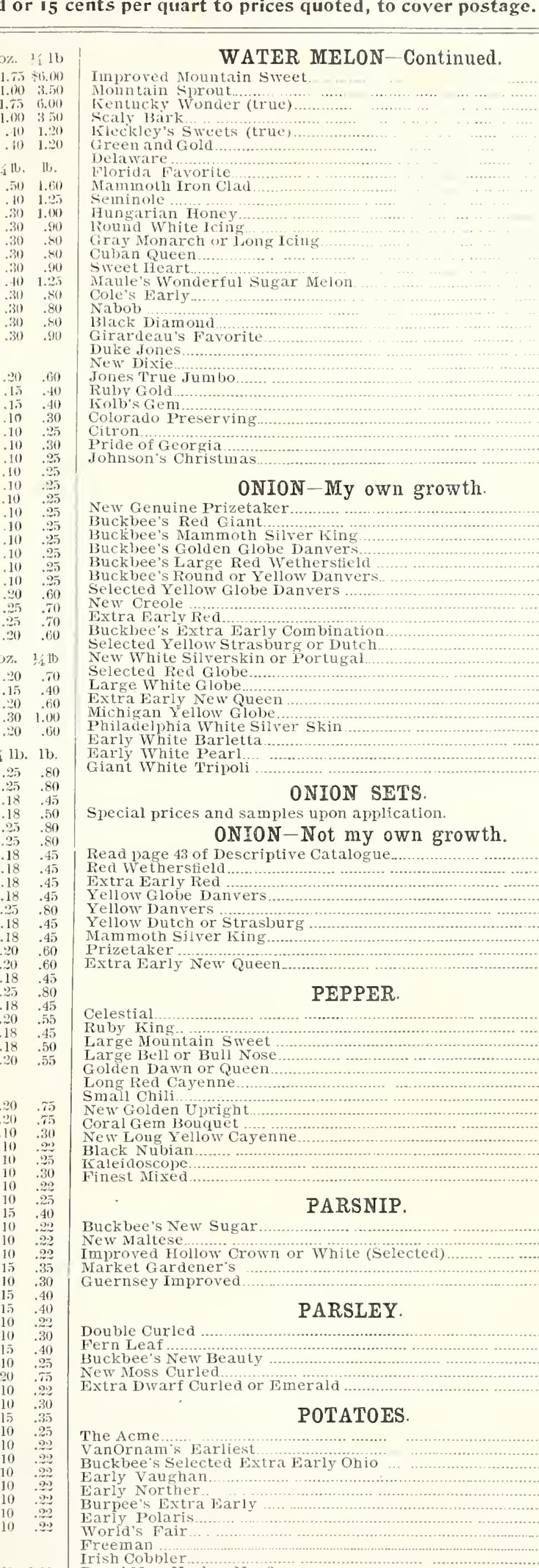

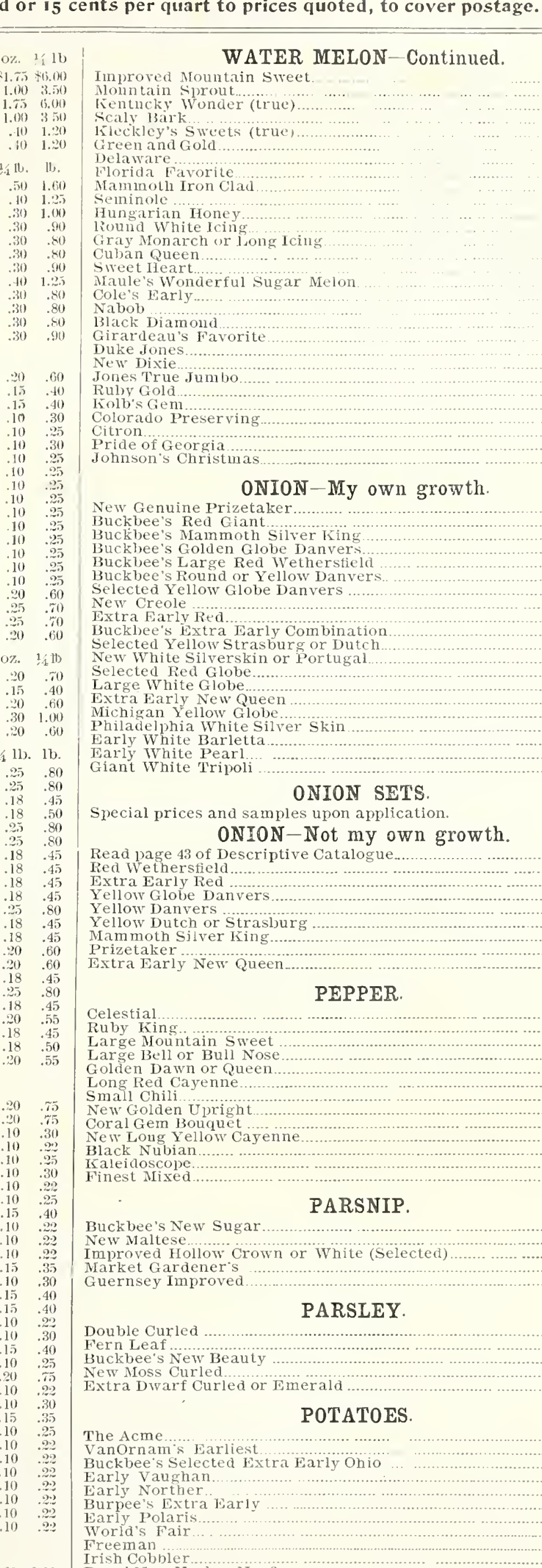

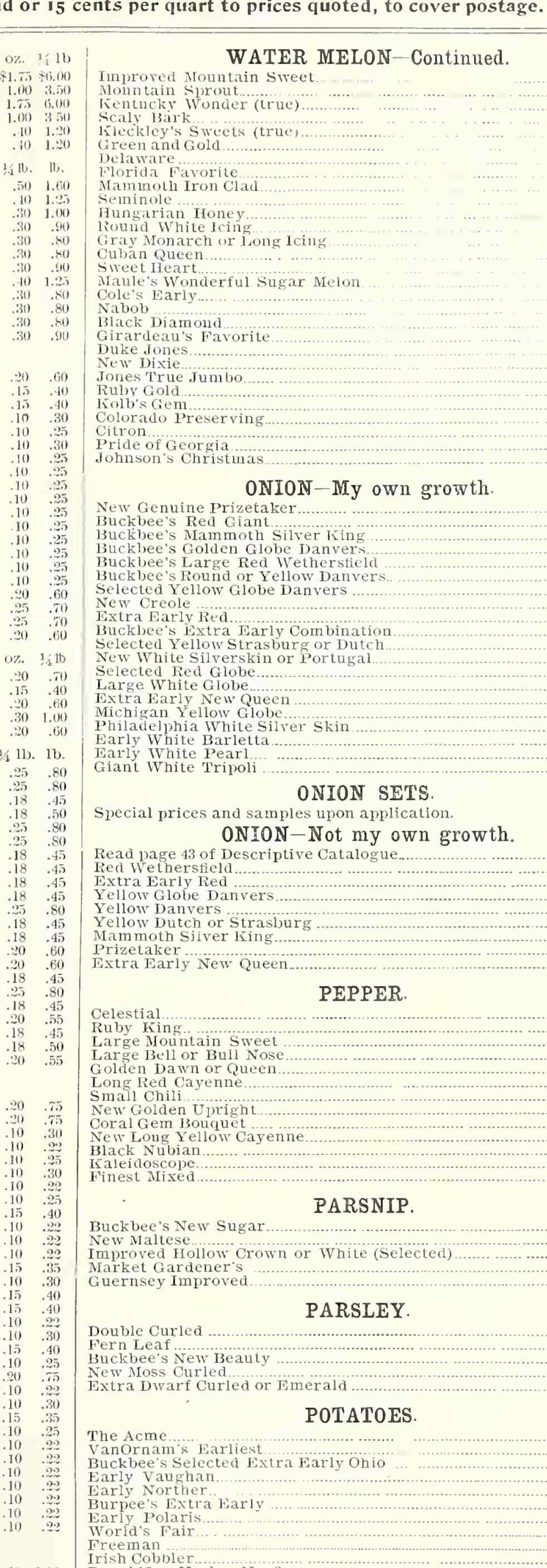

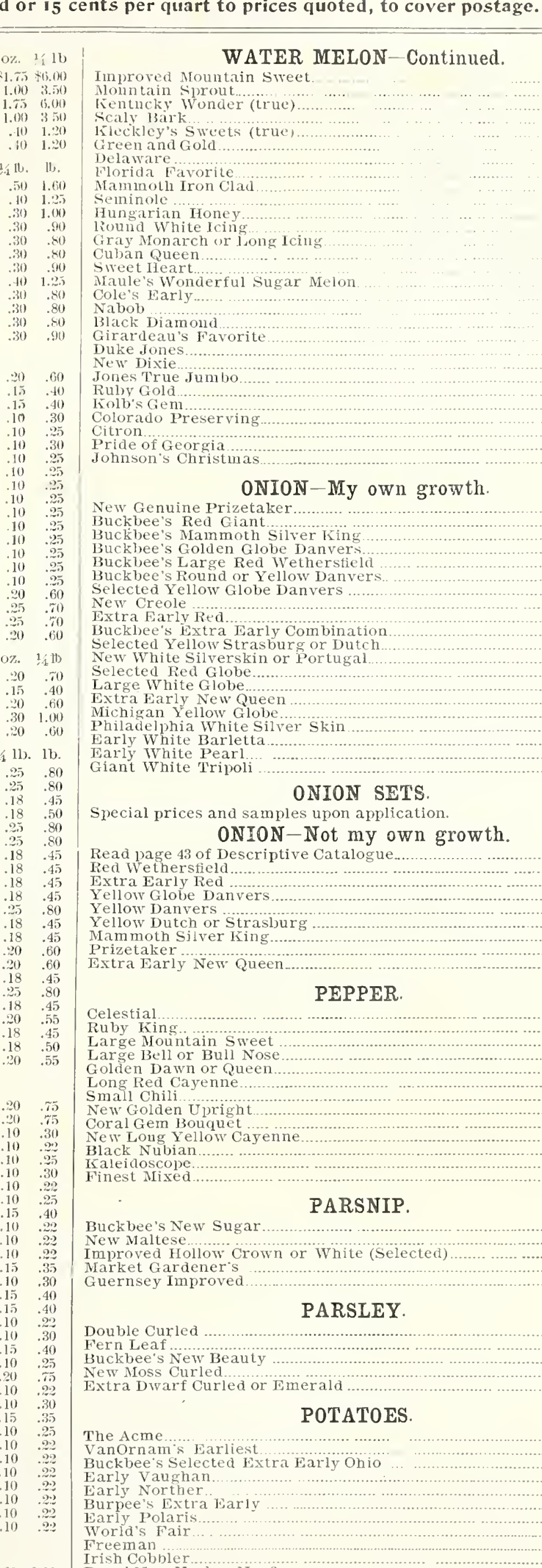

New Genuine Prizetaker

uckbee's Mammoth Silver Kin

thetherstiel

ow Strasburg or Dutc.

xtra Early New Quee

hiladelphia White Silver Ski

Carly White Barlett

Giant White Pripol.

ONION SETS

ONION-Not my own growth

Red Wethersfield

.

.

Prizetale

PEPPER

oz. $\quad 1 / 4$ tb

$15 \quad .50$

$.15 \quad .40$

$\begin{array}{ll}.15 & .40 \\ .15 & .40\end{array}$

$.15 \quad .40$

$\begin{array}{ll}15 & .50 \\ 20 & .60\end{array}$

$\begin{array}{ll}25 & .75 \\ 20 & .60\end{array}$

$\begin{array}{ll}20 & .50 \\ .20 & .50 \\ .50 & .50\end{array}$

.50

Ib. $1 \mathrm{~b}$.

$20 \quad .60$

$10 \quad .20$

$15 \quad .35$

$\begin{array}{ll}15 & .50 \\ .00 & .75 \\ 15 & .50\end{array}$

15. $\quad .50$

$13 \mathrm{bl}$.

Freeman ....

Irish Cobbler...............

Great Divide

Anerican

Carman No...........

Carman

Write for special prices in quantity
3.00
3.00

3.00

2.50
2.75
2.75

2.55
2.50
2.75

2.75
3.00

55
55
55
50
55
75
95
80
10
0
0
0
0
0
0
0
0
5
0
0
0
0
0
.5
5
5

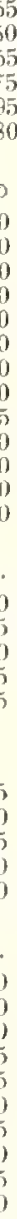

3.00
250

2.50

2.75
3.00
3.00 
If wanted by mail, add Io cents per pound or 15 cents per quart to prices quoted, to cover postage.

\begin{tabular}{|c|c|c|c|c|c|}
\hline SWEET POTATOES. & & Bbl. & SQUASH. & ${ }^{1}, 1$ & \\
\hline 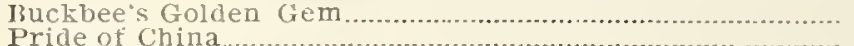 & & $\$ 4.00$ & Buckbee s Giant Summer Croo & $8.10 \leqslant$ & \\
\hline $\begin{array}{l}\text { Pride of China............. } \\
\text { Western Beauty........ }\end{array}$ & & $\begin{array}{l}4.00 \\
4.00\end{array}$ & $\begin{array}{l}\text { Buckbee s New Mammoth Whit } \\
\text { Buckbee's Mammoth Yellow B }\end{array}$ & .10 & \\
\hline Yellow Jersey ............ & & 2.50 & $\begin{array}{l}\text { Buck Dee s Mammoth Yellow Bu } \\
\text { Summer Crookneck }\end{array}$ & .10 & \\
\hline Yellow Nansemond... & & 2.50 & Early White Bush & .10 & \\
\hline $\begin{array}{l}\text { Red Jersey } \\
\text { Red Bermuda }\end{array}$ & & $\begin{array}{l}3.00 \\
3.00\end{array}$ & $\begin{array}{l}\text { Early Yellow Bush } \\
\text { Pineapple........ }\end{array}$ & .10 & \\
\hline 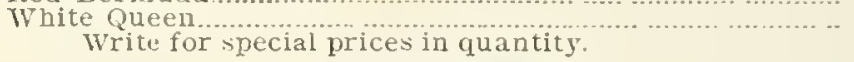 & & 3.00 & $\begin{array}{l}\text { Perfect Gem } \ldots \ldots \ldots \ldots \ldots \ldots \ldots \ldots \\
\text { Cocozelle }\end{array}$ & -.111 & \\
\hline PUMPKIN. & $\frac{1}{4} 1 \mathrm{lb}$. & b. $1 \mathrm{~b}$. & $\begin{array}{l}\text { Pike's Peak, or Sibley } \\
\text { New Delicata }\end{array}$ & .10 & \\
\hline Buckbee's Sandwich Island & .60 & $\therefore .00$ & Der Wing ....... & 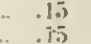 & \\
\hline New Calhoun & .15 & & Chilian Giant & .15 & \\
\hline (2) & .15 & .35 & New Turban or Essex Hybrid & .10 & \\
\hline han ...................................... & .15 & & Bay State & .10 & \\
\hline Quaker Pie & .15 & & $\begin{array}{l}\text { Cocoanut .......................... } \\
\text { Prolific Orange Marrow }\end{array}$ & .10 & \\
\hline $\begin{array}{l}\text { King of the Mammoth: } \\
\text { lese Pie........ }\end{array}$ & .20 & .75 & Improved Boston MIarrow. & -.10 & \\
\hline $\begin{array}{l}\text { nese Pie } \\
\text { Sweet Potato..... }\end{array}$ & .15 & & The Faxon. & $\ldots$ & \\
\hline $\begin{array}{l}\text { e sweet Potato... } \\
\text { theese .................. }\end{array}$ & .10 & .30 & & .1 .15 & \\
\hline ….... & .10 & .30 & New Fordhook & .10 & \\
\hline Mi & .15 & & New Chicago Warted Hub & .15 & \\
\hline $\begin{array}{l}\text { Buckbee's Western Field ( } 1 / \text { bu. 60c. } \\
\text { Buckbee's Pure Gold Field (1/bu. } \%\end{array}$ & & .10 & Improved Hubbard ............ & .10 & \\
\hline $\begin{array}{l}\text { Buckbee's Pure Gold Field (1. bu. } \\
\text { Jumbo or Mammoth Potiron.......... }\end{array}$ & .15 & $\begin{array}{l}.15 \\
.35\end{array}$ & TOMATC & & \\
\hline Mammoth Tours .............. & .15 & .35 & st Market... & & \\
\hline $\mathrm{RA}$ & & & Favorite & .30 & \\
\hline Buc & .15 & .50 & Royal Red. & .30 & \\
\hline Forcing ......... & .15 & .50 & $\mathrm{Ne}$ & .30 & \\
\hline (n..................... & .25 & .75 & rimson cushio & & \\
\hline 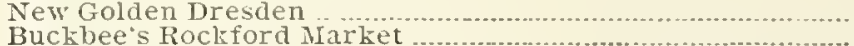 & .15 & .50 & rimson Cushio & .60 & \\
\hline (n) & .10 & .75 & $\mathbf{I}$ & .35 & \\
\hline (n) & .10 & .20 & $\mathbf{E a}$ & & \\
\hline & .10 & .28 & $\mathrm{Li}$ & $\begin{aligned} 30 \\
30\end{aligned}$ & \\
\hline …... & .10 & .2 & & .30 & \\
\hline ........ & .10 & $\therefore$ & erfe & .30 & \\
\hline (.................. & .1 & & & .30 & \\
\hline - & .1 & $\therefore$ & & .40 & \\
\hline 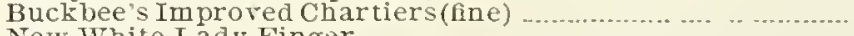 & .1 & $\therefore$ & or No. 400 & .60 & \\
\hline (n) & .10 & & $\because \mathrm{Hrbr}$ & .30 & \\
\hline (n) & .10 & $\therefore$ & er's 1 & .30 & \\
\hline & .1 & & & & \\
\hline$\ldots . . . .$. & .1 & .2 & & .30 & \\
\hline & .1 & .3 & 6 & & \\
\hline & .1 & & & .30 & \\
\hline ......... & .1 & & & .40 & \\
\hline ........... & .1 & $\therefore$ & & . 40 & \\
\hline …....- & .1 & 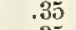 & 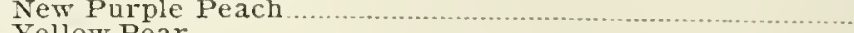 & .40 & \\
\hline & .1 & & & .40 & \\
\hline (n)................... & .1 & $\therefore$ & & .40 & \\
\hline n....... & .1 &. & & .40 & \\
\hline .......... & .1 & & & & \\
\hline ..................... & .15 & $: 4$ & kbee's Sn & (4) & \\
\hline $\begin{array}{l}\text { Winter Radishes Mixed } \\
\text { Early Deep Scarlet Turnip }\end{array}$ & $\begin{array}{l}.15 \\
.10\end{array}$ & $\begin{array}{l}.10 \\
.30\end{array}$ & Mised $\mathbf{H}$ & .6 & \\
\hline SALSIFY, OR VEGETABLE OYSTER. & & & & & \\
\hline & .15 & .50 & & .10 & \\
\hline & .2 & & & .10 & \\
\hline Buclabee's All America. & .30 & 1.00 & & .10 & \\
\hline SPIN. & & & - & & \\
\hline in & & & gue ............ & & \\
\hline ... & & .1 & Extra Early Purple Top Milan & .15 & \\
\hline …....... & & $.1 \%$ & RUTA BAGAS. & & \\
\hline ed Thick Le: & & .12 & 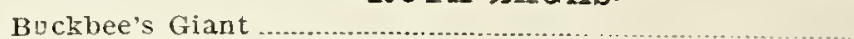 & & \\
\hline idin & & .1 & El & .10 & \\
\hline Prickly or Wint & & .1 & & .10 & \\
\hline Monstrous Viroflay.. & & .12 & Buckhee's Mammoth White Russian & .10 & \\
\hline
\end{tabular}

Long standing Pror Winter.
Prickly or Winstrous Viroflay

\section{MISCELLANEOUS.}

Borecole or hale, Tall Green Curled Scotch Half Dwarf German Ioss Curled Malf Dwarf German Moss Early Purple Cape

Broccol. Early White Cape................

Caper,

cardoon

Chervil, Curled.

Chicory, Large Rooted or Coffee

Collards True Geor.

Corn Salad or Fetticus. Improved Large Seeded

Cress, Fine Curled or Peppergrass True Upland

Dandelion, Improved Jarge Leaf

En "Common.

Few Green Curle

New Moss Curled.............

Broad Leave
White curled

Gaurds-Special Prices upon application

Herbs-Special Prices upon application.

Horse Radisn Sets, 60c. per $100, \$ 5.00$ per 1,000

IKale, see Borecole.

Kohl Rabi, Early White Vienna. Early Purple Vienna.

Leek, Large London Flag...

Rouen Large Winter Large Musselburg.

Mango Melon, or Vegetable Peach

Martynia. Proboscidea for picklin

年 Best

Black or Brown Seeded

Southern Giant Curled

Nasturtium, Tall Mixed

Okra, Improved White Velvet

New Lady Finger

Dwarf Gieen Improred

Long Green

Tall or Long White

Peanuts Tew Mammoth rireinia

Rhubarb, Buckbee's Western Market

$$
\text { Linnaeus }
$$

Phu

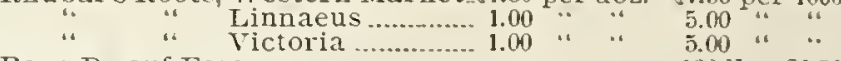

